\title{
Multiharmonic rf feedforward system for beam loading compensation in wide-band cavities of a rapid cycling synchrotron
}

\author{
Fumihiko Tamura, ${ }^{*}$ Masanobu Yamamoto, Chihiro Ohmori, Alexander Schnase, Masahito Yoshii, \\ Masahiro Nomura, Makoto Toda, Taihei Shimada, Keigo Hara, and Katsushi Hasegawa \\ J-PARC Center, KEK \& JAEA, 2-4 Shirakata-Shirane, Tokai, Naka, Ibaraki, Japan 319-1195
}

(Received 30 March 2011; published 31 May 2011)

\begin{abstract}
For the acceleration of very high intensity protons in the $3 \mathrm{GeV}$ rapid cycling synchrotron (RCS) of the Japan Proton Accelerator Research Complex (J-PARC), the beam loading compensation in the rf accelerating cavities is implemented. The magnetic-alloy (MA) loaded cavities, which have a wideband frequency response, are employed in the J-PARC RCS. The wake voltage in the wide-band MA cavity consists of not only a component of the fundamental accelerating rf, but also the higher harmonics. We developed a multiharmonic feedforward compensation system, which handles the most important three harmonics $(h=2,4,6)$. The function of the multiharmonic feedforward is described. Adjustments of the amplitude and phase patterns for the cancellation of the wake voltage during the acceleration period are not trivial. We developed the commissioning methodology of the feedforward. We describe the methodology in the cases without and with driving the accelerating rf. The commissioning of the systems for 11 cavities in the RCS was successful. We describe the commissioning results and the beneficial effects of the feedforward compensation in the beam operation.
\end{abstract}

DOI: 10.1103/PhysRevSTAB.14.051004

PACS numbers: 29.20.- c, 29.27.-a

\section{INTRODUCTION}

In the $3 \mathrm{GeV}$ rapid cycling synchrotron (RCS) of the Japan Proton Accelerator Research Complex (J-PARC) [1-3]. the magnetic-alloy (MA) loaded cavities are employed to generate the high accelerating voltage, which is necessary to accelerate high intensity proton beams. The MA-loaded cavity can achieve a high accelerating field gradient in the order of $20 \mathrm{kV} / \mathrm{m}$, which is 2 times higher than the field achieved by ferrite-loaded cavities $(10 \mathrm{kV} / \mathrm{m})$. The parameters of the J-PARC RCS and its rf system are listed in Table I. In the RCS, the maximum accelerating voltage is $400 \mathrm{kV}$ with 11 cavities.

The $\mathrm{Q}$ value of the MA cavity is set to 2 . This wide frequency response covers the frequency sweep to follow the velocity change during acceleration without the tuning bias loop which is necessary in case of ferrite cavities. The low-level rf (LLRF) control system for the MA cavities is more simple than that for ferrite cavities.

Furthermore, the wide-band frequency response of the MA cavity $(Q=2)$ covers the frequency range of the second harmonic $(h=4)$. In the dual-harmonic operation, each cavity is driven by the superposition of the fundamental accelerating harmonic $(h=2)$ and the second harmonic $(h=4)$ for bunch shaping. By the dual-harmonic

\footnotetext{
*fumihiko.tamura@j-parc.jp

Published by the American Physical Society under the terms of the Creative Commons Attribution 3.0 License. Further distribution of this work must maintain attribution to the author(s) and the published article's title, journal citation, and DOI.
}

operation, a large amplitude second harmonic rf can be generated without extra second harmonic cavities. Because of the limited circumference of the RCS, the dualharmonic operation is essential to generate the large amplitude second harmonic rf. Applying the large amplitude second harmonic rf during the injection period, the bunching factor, which is defined by the ratio of average and peak current, is improved [4] and the beam losses due to the space-charge effects are reduced well, from $7 \%$ to less than $1 \%$ for $300 \mathrm{~kW}$ equivalent beam $\left[2.5 \times 10^{13}\right.$ protons per pulse (ppp)] [5].

The beam loading [6] is a key issue of high intensity proton rings. The relative loading factor $Y$,

$$
Y=I_{b} / I_{g}
$$

TABLE I. Parameters of the J-PARC RCS and its rf system.

\begin{tabular}{|c|c|}
\hline Circumference & $348.333 \mathrm{~m}$ \\
\hline Energy & $\begin{array}{c}\text { (design) } 0.400-3 \mathrm{GeV} \\
\text { (present) } 0.181-3 \mathrm{GeV}\end{array}$ \\
\hline Beam intensity & $\begin{array}{c}\text { (design) } 8.3 \times 10^{13} \mathrm{ppp} \\
\text { (achieved) } 3.3 \times 10^{13} \mathrm{ppp}\end{array}$ \\
\hline Accelerating frequency & $0.938-1.671 \mathrm{MHz}$ \\
\hline Harmonic number & 2 \\
\hline Maximum rf voltage & $\begin{array}{l}\text { (design) } 450 \mathrm{kV} \\
\text { (achieved) } 400 \mathrm{kV}\end{array}$ \\
\hline Repetition & $25 \mathrm{~Hz}$ \\
\hline Number of cavities & $\begin{array}{l}\text { (design) } 12 \\
\text { (installed) } 11\end{array}$ \\
\hline $\mathrm{Q}$ value of rf cavity & 2 \\
\hline
\end{tabular}


where $I_{b}$ is the beam current and $I_{g}$ is the generator current, is a convenient parameter to consider how severe the beam loading effects are to the rf feedback system. It is recommended that $Y$ should be less than 1. MA cavities have relatively low shunt resistances compared to ferrite cavities and the generator currents are comparatively large, which is a benefit to handle the beam loading. In the RCS design, the relative loading factor $Y$ is almost always kept less than 1 at the design beam power, $1 \mathrm{MW}$, except near the extraction, where the rf voltage is small to match the rf bucket of the RCS with that of the main ring and there $Y$ is fairly higher than 1 . Thus, the beam loading compensation is necessary to accelerate the beams at the full intensity.

Furthermore, due to the wide-band frequency response, the wake voltages consist not only of the fundamental accelerating harmonic but also the higher harmonic components. The rf bucket distortion is an issue for high power beam acceleration. A longitudinal particle tracking simulation [7] has shown that at least the fundamental and second harmonic components must be compensated to accelerate the full-intensity beams.

We employ the rf feedforward (FF) method to compensate the multiharmonic beam loading. The beam loading compensation by the feedforward method was utilized in various proton rings, for example, KEK-PS [8,9], CERN-PS [10,11], and ISIS [12]. In all of them, ferrite cavities are used in single harmonic operation.

The multiharmonic beam loading compensation in a wide-band MA cavity by the feedforward method was tested by using electron beams [13]. Also in the barrier cavity in the Brookhaven AGS, which has a very low Q value about 0.6 , a multiharmonic FF system was used [14]. It is reported in the references above that the multiharmonic beam loading compensation by the rf feedforward method works; however, the compensation was performed without driving rf voltages and frequency sweeps, which is necessary for the application to the J-PARC RCS.

All of the rf feedforward systems described above are implemented by analog circuits. It is not easy to adjust the feedforward parameters during a full acceleration period, in which the parameters such as frequencies and relative loading factors $Y$ vary, by analog circuits.

We developed a multiharmonic rf feedforward system as a part of the LLRF control system for the J-PARC RCS. The LLRF control system including the feedforward is fully implemented by digital circuits to realize the stable and reproducible control. In Sec. II we describe the overview of the multiharmonic rf feedforward system.

The feedforward method requires delicate adjustment of the parameters, while the hardware implementation itself is simple [15]. We established the commissioning methodology of the multiharmonic feedforward. We describe the details of the methodology and present the commissioning results.

\section{MULTIHARMONIC FEEDFORWARD SYSTEM}

\section{A. Overview of LLRF control system}

A simplified block diagram of the LLRF control system of the J-PARC RCS is shown in Fig. 1 [16]. All LLRF modules are implemented by digital circuits, which are working with a system clock at the frequency of $36 \mathrm{MHz}$ generated by locking to the $12 \mathrm{MHz}$ master clock of the J-PARC timing system [17], to synchronize to the other accelerator devices in the J-PARC complex.

The multiharmonic phase reference signals are generated in the "standard-phase generator (SPG)" and the "beam-current analysis (BCA)" modules by the direct digital synthesis (DDS) technology [16]. In the SPG and BCA modules, the phase reference signals of the harmonics $(h=2,4)$ and $(h=2,4,6)$ are generated, respectively. The SPG and BCA modules contain phase accumulators, which accumulate phase increments corresponding to the revolution frequency at each system clock, so that sawtooth phase reference signals are generated. The phase reference signals of the higher harmonics of the revolution are generated by multiplying the revolution phase signal by the harmonic number $h$. Since the multiharmonic phase reference signals are generated without phase locked loop, the synchronization between the harmonics is guaranteed. The multiharmonic phase reference signals are used for generation of the driving rf signal $(h=2,4)$ and for detection of the rf voltage and the beam signal by using in-phase/ quadrature-phase (I/Q) demodulation.

By using the DDS, the frequency is highly accurate. The accuracy is in the order of $10^{-7}$, while that of analog voltage controlled oscillators is about $10^{-4}$.

As described in the Introduction, the MA cavity is driven by the superposition of the fundamental and the second harmonic rf signals. To control the amplitude of both harmonics, the dual-harmonic auto voltage control (AVC) is implemented [18]. By the strong control of the dualharmonic AVC, the amplitudes of the harmonics are maintained as programmed under the high power beam load at the beam power of $300 \mathrm{~kW}$. The dual-harmonic AVC is implemented in the "RFG (rf generator)" module.

The common beam feedbacks, the phase and radial feedbacks, are implemented in the LLRF control system of the RCS. However, the radial feedback is not used in the beam operation, because the rf frequency of the DDS and the bending magnet fields are very stable and reproducible. When required, for example, at startup time after a shutdown period, we take an orbit signal of a full accelerating cycle and correct the frequency pattern [19]. Thus, the rf and beam revolution frequencies are highly reproducible. The phase feedback of the fundamental harmonic damps longitudinal dipole oscillations, and that of the second harmonic locks its phase to the phase of the fundamental harmonic so that the desired voltage waveform is obtained [20]. 


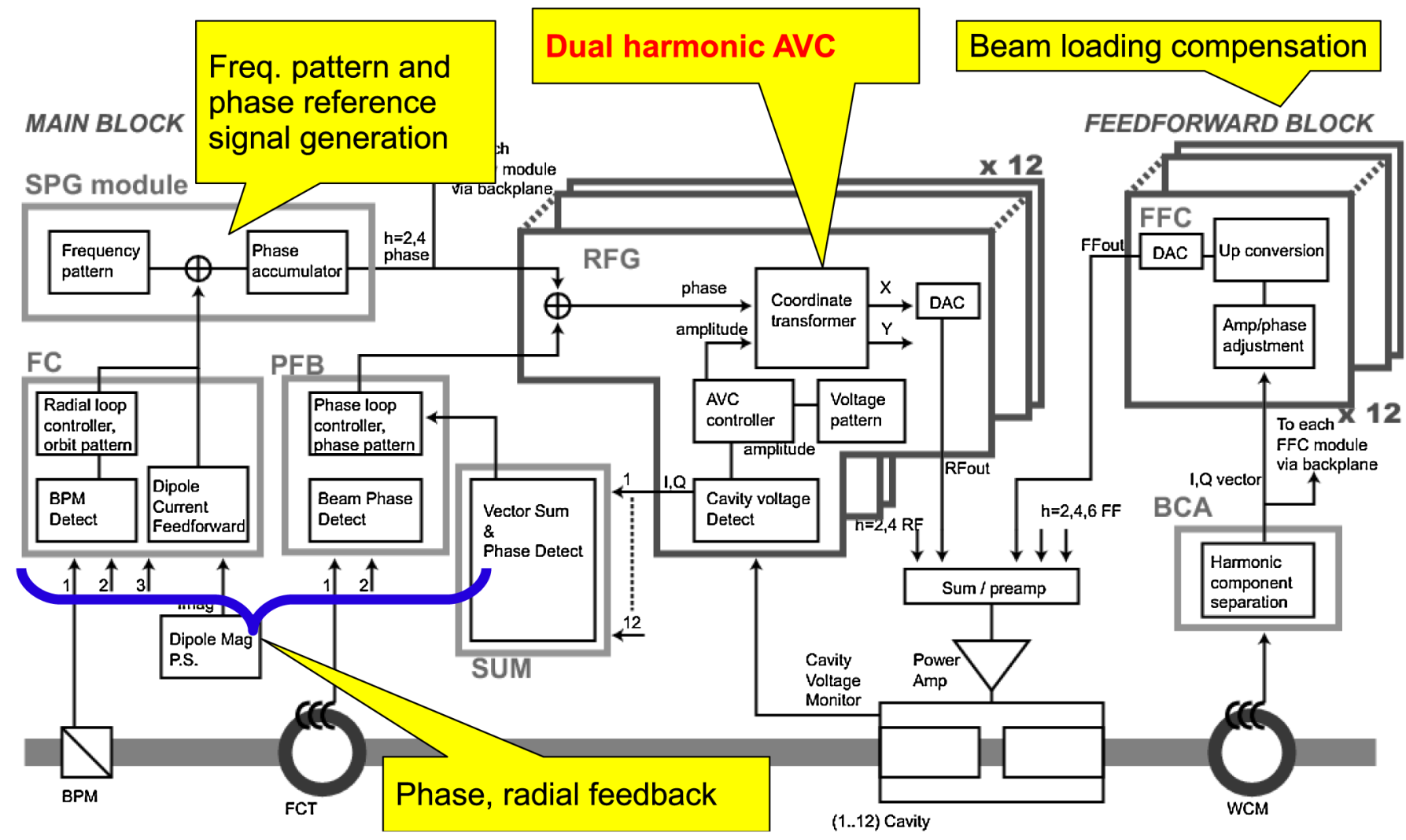

FIG. 1. Simplified block diagram of the RCS LLRF control system.

The rf feedforward system uses the wall current monitor (WCM) signal to generate the feedforward compensation signal. The feedforward signal is generated for each of the cavities and summed up to the driving rf (RFG output) signal by the summation amplifier. The function of the rf feedforward system is described in the following subsection.

\section{B. Function of the feedforward system}

The operational principle of the rf feedforward method is shown in Fig. 2. Assuming that the beam current is $i_{\text {beam }}$, if the feedforward system generates $-i_{\text {beam }}$ in the cavity by using the WCM beam signal in addition to the driving rf

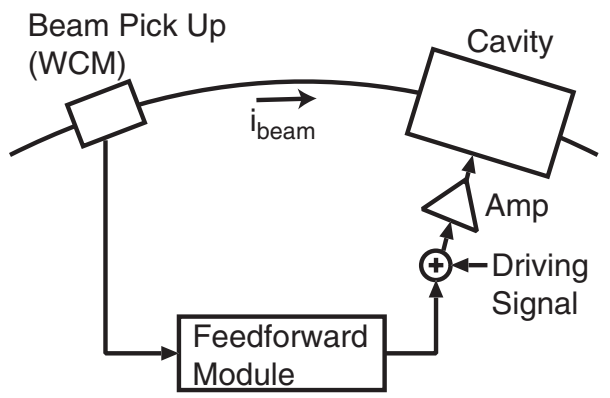

FIG. 2. A conceptual diagram of the rf feedforward method. The feedforward module generates $-i_{\text {beam }}$ so that the wake voltages are canceled. current for the rf voltage generation, the wake voltage is canceled. As described in the previous section, the wake voltage consists not only of the fundamental accelerating harmonic but also the higher harmonics. We developed the multiharmonic feedforward system, which can compensate the beam loading of the most important three harmonics $(h=2,4,6)$.

The block diagram of the feedforward system is shown in Fig. 3. The beam signal from the WCM is digitized by an analog-to-digital converter. The digitized beam signal is led into the I/Q detection blocks for the harmonics $(h=2,4,6)$. Sine and cosine signals with unity amplitude of the selected harmonic are generated by using the phase reference signal of the harmonic, and the beam signal is multiplied by the sine and cosine signals. Applying the low-pass filter, the I/Q vectors of the selected harmonic, $\left(I_{\text {out }}, Q_{\text {out }}\right)$ are obtained as

$$
\begin{aligned}
I_{\text {out }} & =A(h, t) \sin [\phi(h, t)], \\
Q_{\text {out }} & =A(h, t) \cos [\phi(h, t)],
\end{aligned}
$$

where $h$ is the harmonics selected, $A(h, t)$ and $\phi(h, t)$ are the amplitude and the phase of the selected harmonics, respectively. This function is implemented in the BCA (beam-current analysis) module in Fig. 1. The I/Q vectors of the harmonics are distributed to the feedforward control 


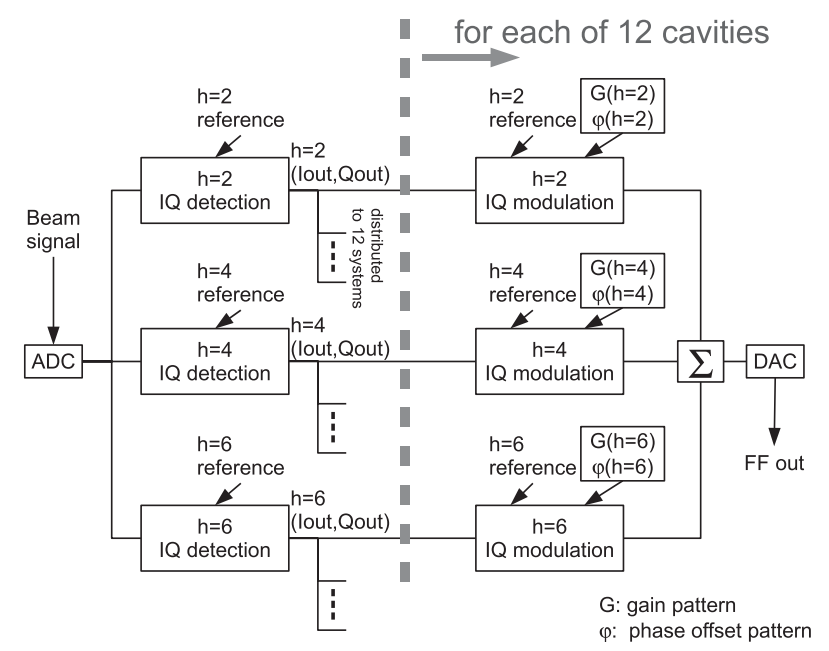

FIG. 3. Block diagram of the multiharmonic rf feedforward system.

(FFC) modules to generate the compensation signals for 12 cavities.

To generate the compensation signal, the I/Q modulation technique is employed. Like the I/Q detection, the sine and cosine signals are generated by using the phase reference signal of the selected harmonic, and the I/Q vector and the sine and cosine signals are multiplied so that the I/Q modulation output is obtained as

(I/Q modulation output)

$$
\begin{aligned}
& =G\left[I_{\mathrm{out}} \cos \left(\omega_{h} t+\psi\right)+Q_{\mathrm{out}} \sin \left(\omega_{h} t+\psi\right)\right] \\
& =G A(h, t) \sin \left[\omega_{h} t+\phi(h, t)+\psi\right],
\end{aligned}
$$

where $G$ and $\psi$ are the gain and phase patterns and $\omega_{h}$ is the angular frequency of the selected harmonic, namely, $\omega_{h}=h \times \omega_{\text {rev }}$ (revolution frequency).

Finally, the I/Q modulation outputs are summed up and the feedforward signal is converted by the digital-to-analog converter. The feedforward signal is sent to the summation amplifier.

Essentially, the feedforward system works as a bandpass filter, which has the passbands at the harmonics of $(h=2,4,6)$ with programmed gain and phase patterns. The passbands follow the frequency sweep during the acceleration by referring to the phase reference signals generated by the DDS.

In the feedforward system of the RCS, the pattern sampling frequency is $1 \mathrm{MHz}$ [21].

\section{COMMISSIONING OF THE FEEDFORWARD SYSTEM}

As described in the previous section, the amplitude and phase patterns must be adjusted so that the wake voltages of the harmonics are canceled. We describe the commissioning methodology for two cases, without the driving rf and with the driving accelerating rf.

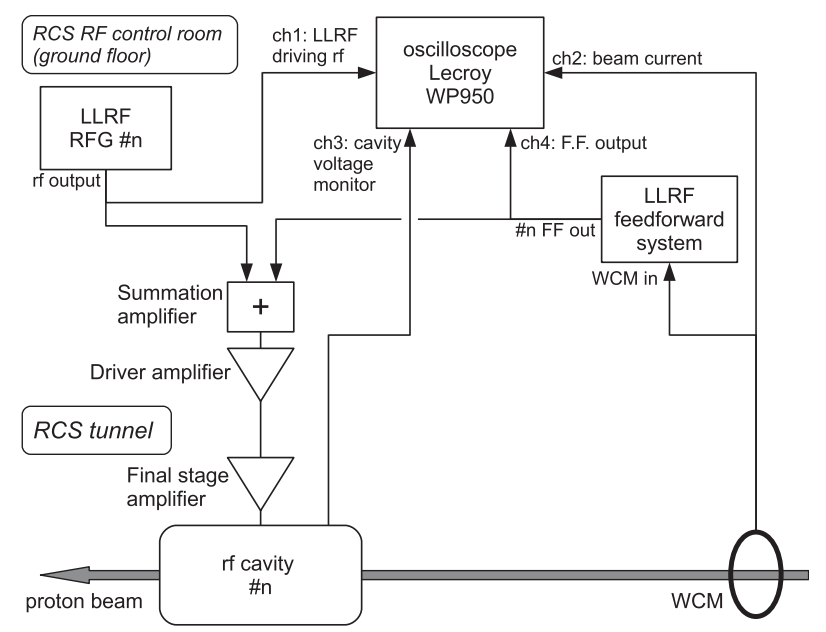

FIG. 4. Beam test setup. The commissioning of the feedforward system is performed for each of the cavities. In the figure, \# $\mathrm{n}$ is the cavity number. The signals are well matched.

The commissioning of the feedforward system was performed with a $300 \mathrm{~kW}$ equivalent beam $\left(2.5 \times 10^{13} \mathrm{ppp}\right)$. The longitudinal painting [4] with the second harmonic rf was fully applied.

The beam test setup is illustrated in Fig. 4. In the figure, $\# \mathrm{n}$ is the cavity number (1-11). The commissioning of the feedforward system is performed for each cavity. The following signals are recorded by the oscilloscope (Lecroy WP950): (i) LLRF driving rf (channel 1); (ii) WCM signal (channel 2); (iii) cavity gap voltage monitor (channel 3); (iv) feedforward output (channel 4).

The signals are recorded at the sampling rate of $200 \mathrm{Ms} / \mathrm{s}$ from the injection to the extraction. The record length is $4.15 \mathrm{M}$ points for each channel.

The harmonic analysis for each channel is performed on the PC and the complex amplitudes of the harmonics $(h=2,4,6)$ are obtained. The complex amplitudes are used for the calculation of the transfer functions and the impedances.

\section{A. Cancellation of the wake voltage without $\mathrm{rf}$ drive}

We describe the commissioning methodology to cancel the wake voltages in a cavity without driving rf voltage. The test was performed to demonstrate the performance of the rf feedforward system, while the situation that one of the cavities is not driven is not a standard operation.

To keep the total accelerating rf voltage, the other cavities were driven by a voltage pattern scaled by a factor 1.1 of the normal voltage pattern. The total idling current of the tube amplifier of the target system was $10 \mathrm{~A}$.

The test was performed for all of the 11 cavities. Here we describe the commissioning method with showing the results of cavity \#10, as follows.

First, the test beam was accelerated without feedforward compensation signal, and the full waveforms of the WCM 

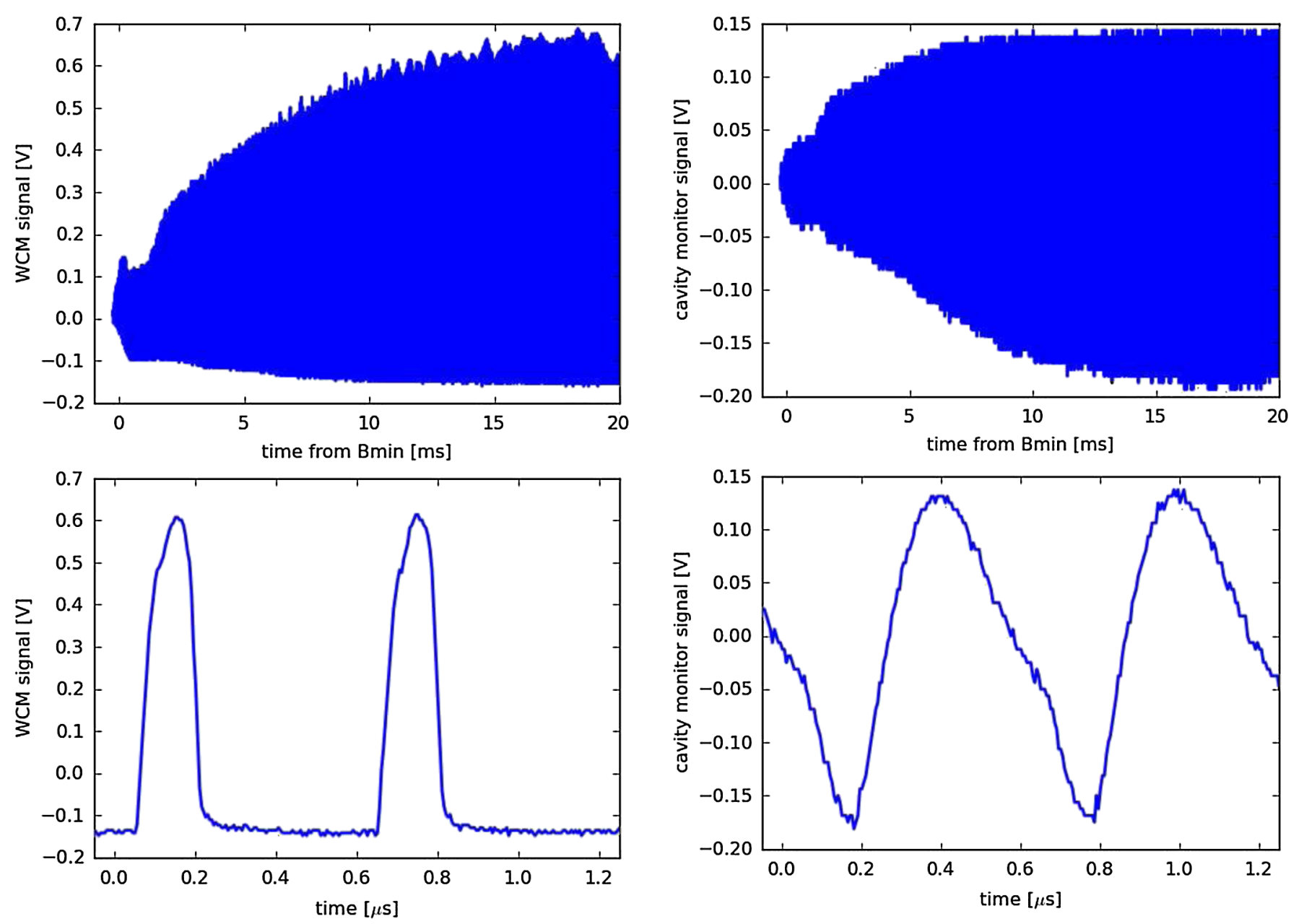

FIG. 5. The WCM signal (channel 2). The envelope of full waveform from the injection to the extraction (upper) and a magnified view near the extraction (lower).

signal (channel 2) and gap voltage monitor of the target cavity (channel 3 ) from the injection to the extraction are recorded. Since the cavity was not driven, the gap voltage consists of only the wake voltage. In Figs. 5 and 6, the recorded signals of the WCM and the gap voltage monitor of cavity \#10 are shown. The harmonic analysis is performed on these signals to extract the $(h=2,4,6)$ complex components.

The amplitudes of the harmonic components of the WCM signal and cavity \#10 gap monitor are shown in Fig. 7. As the revolution frequency increases from the injection to the extraction, the beam current increases. The ratios of the harmonics of the WCM signal correspond to the bunch shape. The second harmonic $(h=4)$ component is very small during the injection period in which the bunch is very flat thanks to the longitudinal painting by using the second harmonic rf voltages [4]. After $2 \mathrm{~ms}$ the second harmonic rf voltage is zero and the second harmonic beam component increases. Since the conversion of the WCM is that

FIG. 6. The gap voltage monitor signal of cavity \#10 (channel 3). The envelope of full waveform from the injection to the extraction (upper) and a magnified view near the extraction (lower). The gap voltage consists of only the wake voltage.

$$
\text { (beam current }[\mathrm{A}])=(\mathrm{WCM} \text { voltage }[\mathrm{V}]) / 0.05 \text {, }
$$

one can see that the $(h=2)$ component of the circulating beam current reaches more than $5 \mathrm{~A}$ just before extraction when the beam of $2.5 \times 10^{13} \mathrm{ppp}$ is accelerated.

Assuming steady state, the wake voltage in frequency domain is expressed as

$$
V_{\text {cav }}(\omega)=Z_{\text {cav }}(\omega) \cdot I_{\text {beam }}(\omega),
$$

where $\omega$ is the angular frequency, $V_{\text {cav }}(\omega)$ the gap voltage, $Z_{\text {cav }}(\omega)$ the cavity gap impedance, and $I_{\text {beam }}(\omega)$ the beam current. The resonant frequency of the cavity is about $1.7 \mathrm{MHz}$. The amplitude of fundamental $(h=2)$ component of the wake voltage increases from the injection to the extraction, not only because the beam current increases, but also because the $(h=2)$ frequency gets closer to the resonant frequency. On the other hand, the second and the third harmonic components $(h=4,6)$ veer away from the resonant frequency. The increments of the amplitudes of the higher harmonics are limited compared to the 

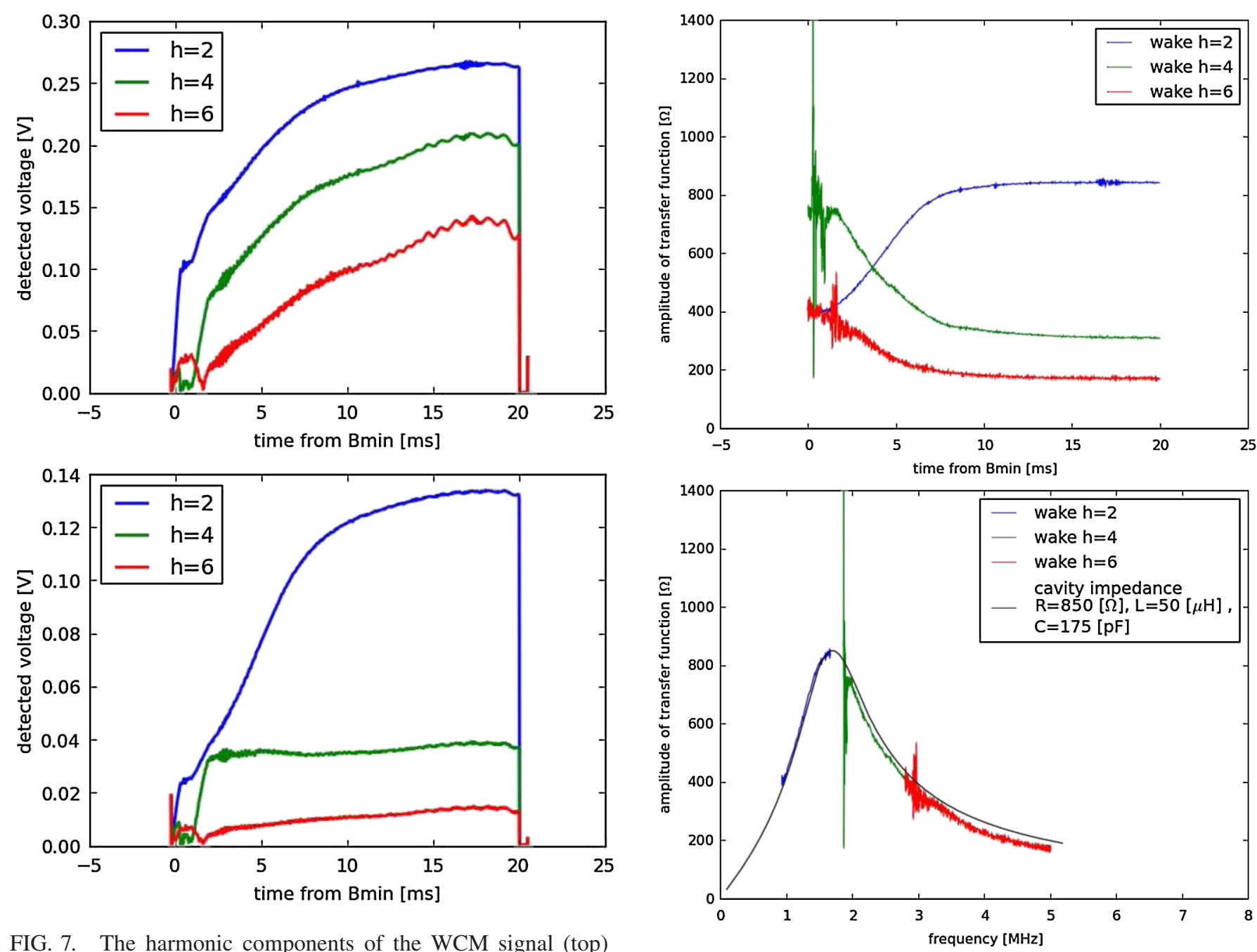

FIG. 7. The harmonic components of the WCM signal (top) and cavity \#10 gap monitor (bottom).

increase of the beam current. By using the conversion of the gap voltage monitor as

$($ gap voltage $[\mathrm{V}])=($ monitor voltage $[\mathrm{V}]) \times 3.35 \times 10^{4}$,

the fundamental component of the wake voltage is more than $4 \mathrm{kV}$ per gap just before the extraction at this beam intensity.

Since the frequency sweep is slow compared to the wide frequency response of the MA cavity $(Q=2)$, a steadystate equation is a good approximation to calculate the time variation of the cavity gap impedance seen by the beam. Equation (6) can be rewritten as

$$
V_{\text {cav }}\left[\omega_{h}(t)\right]=Z_{\text {cav }}\left[\omega_{h}(t)\right] \cdot I_{\text {beam }}\left[\omega_{h}(t)\right],
$$

where $\omega_{h}(t)$ is the angular frequency of the harmonic $(h=2,4,6)$ and $t$ is the time. The frequencies of the harmonics are obtained as functions of time as programmed. To simplify the notation, we define

FIG. 8. Translation to impedance. $Z(\omega)=V(\omega) / I(\omega)$ - (top) as function of time and (bottom) frequency domain.

$$
\begin{aligned}
V_{\text {cav }}\left[\omega_{h}(t)\right] & =V_{\text {cav }}(h, t), \\
I_{\text {beam }}\left[\omega_{h}(t)\right] & =I_{\text {beam }}(h, t), \\
Z_{\text {cav }}\left[\omega_{h}(t)\right] & =Z_{\text {cav }}(h, t) .
\end{aligned}
$$

By using (9), (8) becomes

$$
V_{\text {cav }}(h, t)=Z_{\text {cav }}(h, t) \cdot I_{\text {beam }}(h, t)
$$

By (10) and the complex harmonic components of the beam current and the cavity gap voltage, the cavity impedance is obtained for each harmonics as a function of time. By using the frequency program, one can reconstruct the impedance curve in the frequency domain. In Fig. 8, the gap impedance seen by the beam is plotted. In the figure, the upper plot shows the time variations and the lower shows the reconstructed frequency response. In the lower plot, the frequency response of the parallel circuit model $(R=850 \Omega, L=50 \mu \mathrm{H}, C=175 \mathrm{pF})$ is also plotted. The measured impedance and the circuit model show good agreement. Because the higher harmonic components 
$(h=4,6)$ of the beam current are small at the beginning of the acceleration before $2 \mathrm{~ms}$ as shown in Fig. 7, the impedance calculation shows larger errors due to the small denominators.

The feedforward signal has a delay, due to the long cable lengths from the WCM in the accelerator tunnel to the feedforward system in the ground floor and from the feedforward system to the cavity in the tunnel, and the group delay of the feedforward system. To compensate the delay, the initial phase pattern is advanced from the starting phase as follows:

$$
\Delta f \cdot T_{d} \times 360[\mathrm{deg}]
$$

where $\Delta f$ is the frequency difference of the selected harmonics $(h=2,4,6)$ from that of the injection and $T_{d}$ is the total delay. In our system, the total delay is about $3.6 \mu \mathrm{s}$. A constant gain pattern was used as the initial gain pattern.

Here, the test beam was accelerated with the feedforward compensation signal. With the feedforward signal, the cavity voltage is a superposition of the wake voltage and the feedforward signal. For the harmonics $(h=2,4,6)$, we can write

$$
\begin{aligned}
V_{\text {cav }}(h, t) & =V_{\text {cav, wake }}(h, t)+V_{\text {cav, FF }}(h, t) \\
& =Z_{\text {cav }}(h, t) \cdot I_{\text {beam }}(h, t)+Z_{\mathrm{FF}}(h, t) \cdot I_{\text {beam }}(h, t),
\end{aligned}
$$

where $V_{\text {cav, wake }}(h, t)$ and $V_{\text {cav, FF }}(h, t)$ are the voltage components of the wake and the feedforward signal, respectively. Since $Z_{\text {cav }}(h, t)$ is already known by the previous measurement, $Z_{\mathrm{FF}}(h, t)$, which is the transfer function from the beam current to the feedforward gap voltage, is obtained by using the measured voltage and beam current, $V_{\text {cav }}(h, t)$ and $I_{\text {beam }}(h, t)$.

The impedance seen by the beam is now

$$
Z_{\mathrm{cav}}(h, t)+Z_{\mathrm{FF}}(h, t) .
$$

Assuming the linear response of the feedforward voltage, the gain and phase patterns are modified so that

$$
Z_{\mathrm{FF}}(h, t)=-Z_{\text {cav }}(h, t) .
$$

By the modified pattern, the test beam is again accelerated. Several iterations are necessary to realize the minimum impedance seen by the beam.

The waveform of the gap voltage monitor signal is shown in Fig. 9. Comparing to Fig. 6, one can see that the wake voltage is canceled by the feedforward signal. The harmonic components of the gap voltage monitor are shown in Fig. 10. For all three harmonics $(h=2,4,6)$, the residual voltage is very small compared to the lower plot in Fig. 7.

Finally, the impedance seen by the beam is plotted in Fig. 11. In the figure, the cavity impedances without the
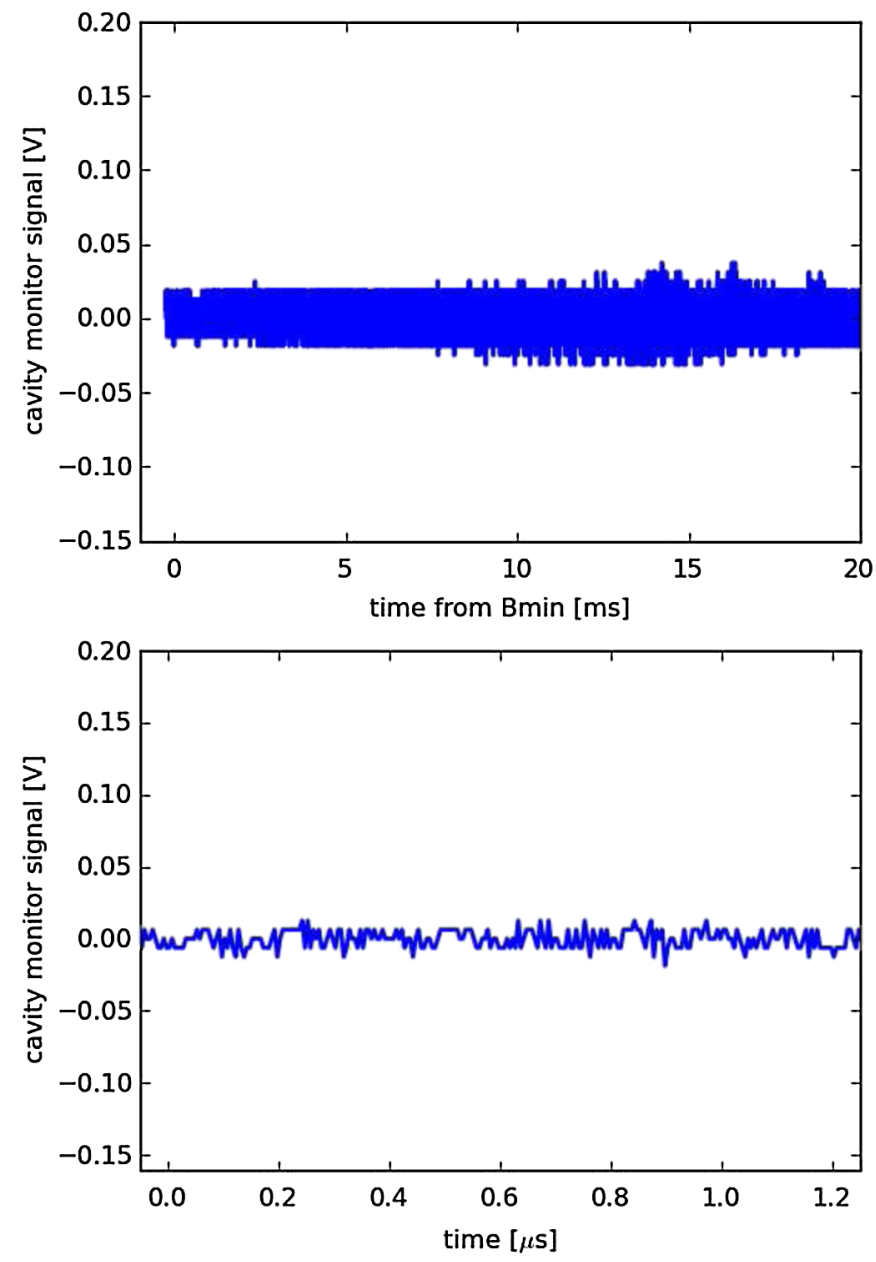

FIG. 9. The gap voltage monitor signal of cavity \#10 (channel 3) with feedforward on. The envelope of full waveform from the injection to the extraction (upper) and a magnified view near the extraction (lower). The wake voltage is canceled and only a noise signal is remaining.

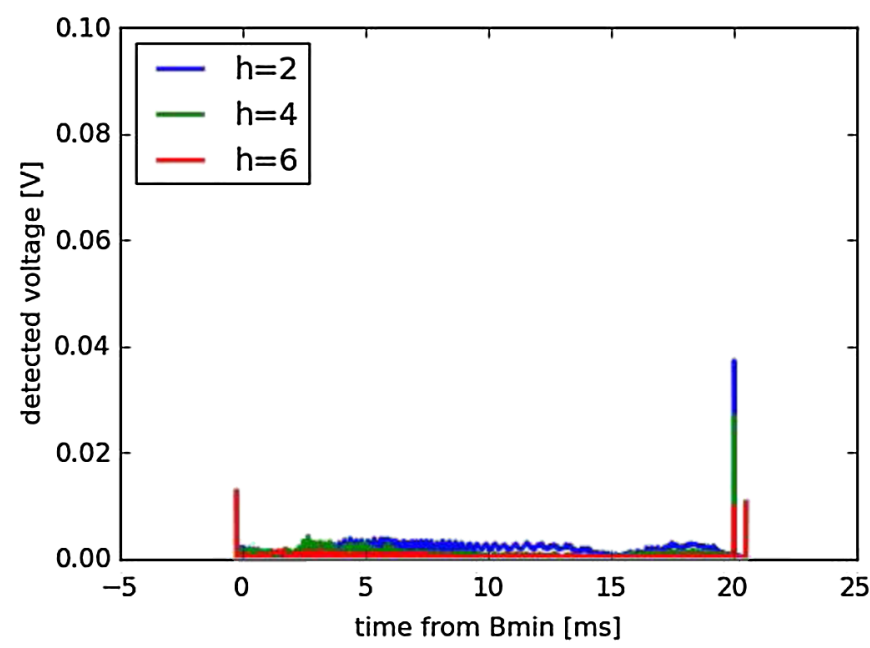

FIG. 10. The harmonic components of the gap monitor signal with feedforward. 


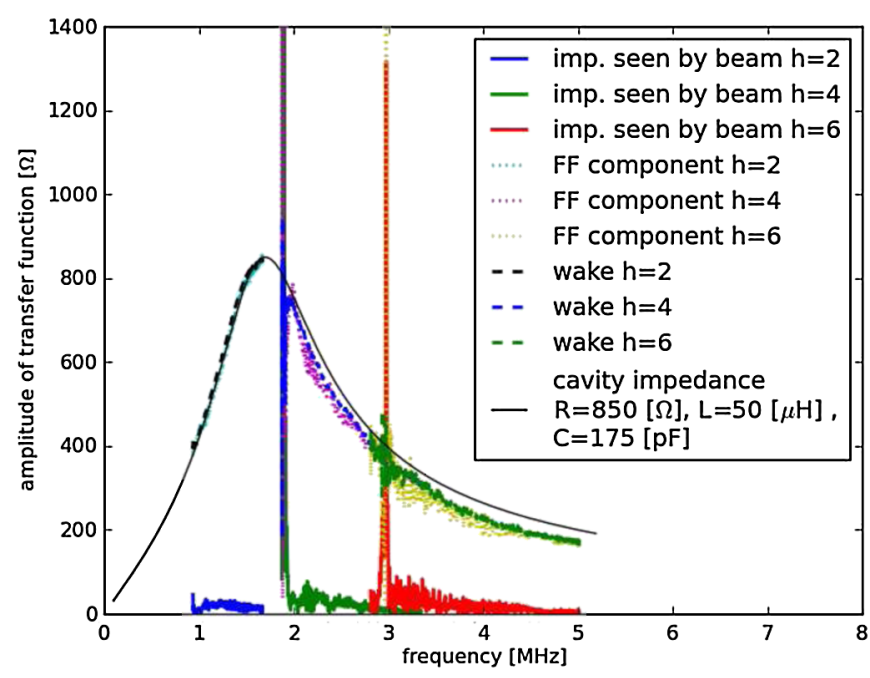

FIG. 11. Comparison of impedance seen by the beam, without and with FF. A reduction of $1 / 10$ in a full accelerating period is achieved for the fundamental accelerating harmonic $(h=2)$.

feedforward $\left(Z_{\text {cav }}\right)$ and the feedforward transfer functions $\left(Z_{\mathrm{FF}}\right)$ are also plotted. Because the pattern is modified so that (14) is satisfied, one can clearly see that the amplitudes of the impedances of the cavity and the feedforward become very close. Thus, the impedance seen by the beam is significantly reduced with the feedforward. For the fundamental $(h=2)$, a reduction in the order of $1 / 10$ from the injection to the extraction is achieved. Except near the injection great reduction is achieved also for the other harmonics $(h=4,6)$.

\section{B. Beam loading compensation with accelerating $\mathrm{rf}$ drive}

We describe the commissioning methodology of the feedforward in a cavity driven by the accelerating $\mathrm{rf}$ voltages.

The gain and phase patterns which are optimum for the case without the driving rf cannot be optimum in the case with the driving $\mathrm{rf}$ too, due to the following reasons. First, the impedances seen by the beam are different between without and with the driving rf, because the tube currents and the parallel resistances of the tubes are different, while the cavity shunt resistance itself is independent of the driving rf. Second, the high power amplifier shows amplitude dependencies. For example, the rf splitter using small ferrite cores in the grid circuit shows a phase shift, which depends on the driving rf amplitude.

The cavity gap voltage is the superposition of the driving rf, the wake voltage, and the feedforward voltage. For the commissioning of the feedforward system, one must separate these components.

We developed the commissioning methodology as described in the following steps.
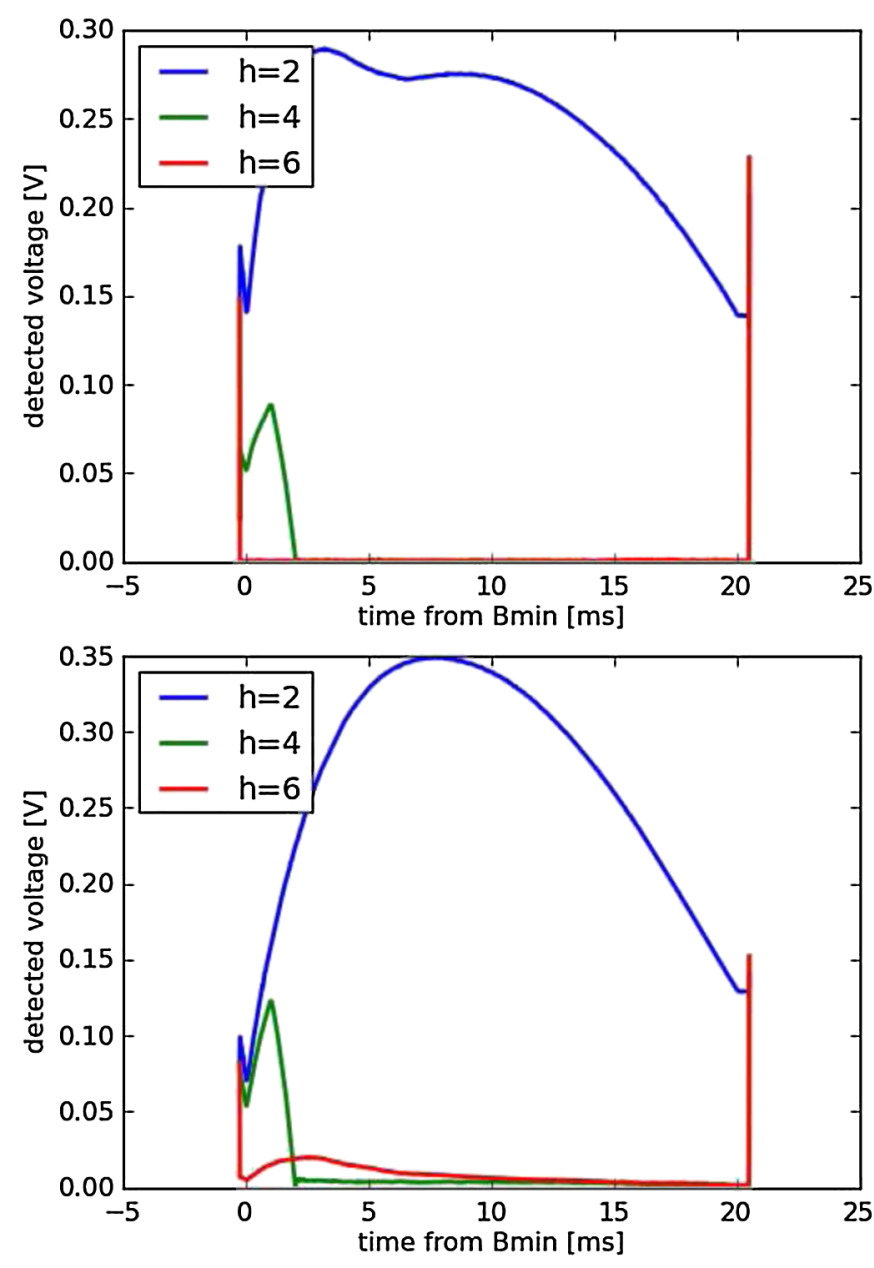

FIG. 12. Step 1 example (cavity \#1). The harmonic components of (top) the LLRF driving rf and (bottom) the cavity voltage are plotted.

\section{Step 1}

First, we obtain the transfer function from the driving rf to the cavity voltage.

The full waveforms of the LLRF driving rf signal and the cavity voltage monitor during the accelerating period without accelerating the beam are recorded. The harmonic analysis for the harmonics $(h=2,4,6)$ is performed on the recorded waveforms. The typical harmonic components of the LLRF driving rf and the cavity voltage monitor are plotted in Fig. 12.

One can notice that the third harmonic $(h=6)$ component appears in the cavity voltage monitor from 0 to $15 \mathrm{~ms}$ while in the LLRF driving rf only the fundamental $(h=2)$ and the second harmonic $(h=4)$ components exist. This is because of the current waveform distortion by the high power tube amplifier driven by the fundamental harmonic rf. In case of high-Q cavities, the third harmonic component is outside of the cavity resonance; however, the voltage of the third harmonic distortion is not negligible in our low-Q $(Q=2)$ cavity. 
Because of the third harmonic component by the distortion, the separation of the wake and the driving if is difficult. In this commissioning the feedforward pattern of the third harmonic $(h=6)$ is not modified by using the measurement Instead a constant gain pattern and the phase pattern obtained by the commissioning without the driving rf is used for $(h=6)$.

By using the complex voltages of the harmonics $(h=2,4)$ of the driving $\mathrm{rf}\left[V_{\mathrm{dr}}(h, t)\right]$ and the cavity voltage $\left[V_{\text {cav }}(h, t)\right]$, the transfer function from the driving rf to the cavity voltage $\left(H_{\mathrm{dr}}^{\text {cav }}(h, t)\right)$ is obtained as

$$
H_{\mathrm{dr}}^{\mathrm{cav}}(h, t)=\frac{V_{\mathrm{cav}}(h, t)}{V_{\mathrm{dr}}(h, t)} .
$$

In the case of the second harmonic $(h=4)$, the voltage program becomes zero during acceleration period, for example, $2 \mathrm{~ms}$ after injection. When the voltage pattern is zero, (15) gives large errors because $V_{\mathrm{dr}}(h, t)$ is very small and contains only noise. To avoid this issue, if $V_{\mathrm{dr}}(h, t)$ is smaller than a certain small threshold, we set $H_{\mathrm{dr}}^{\mathrm{cav}}(h, t)=0$.

\section{Step 2}

As described above, the impedance seen by the beam depends on the tube currents. In this step, we obtain the impedance of the target cavity under the condition of the tube currents with the standard accelerating voltage patterns.

To avoid the variation of the condition, this measurement is performed without the AVC loop. We set a special pattern, which generates similar voltages without AVC to the voltages with AVC.

A test beam is accelerated with the AVC off. The LLRF driving rf voltage (channel 1), the WCM (channel 2), and the cavity gap voltage monitor (channel 3) are recorded for the full accelerating period. The harmonic analysis is performed with the recorded signals.

In Fig. 13, the harmonic components of the WCM beam current and the cavity voltage monitor are plotted. One can notice that the cavity voltage is modified by the wake voltages, especially the fundamental accelerating harmonic $(h=2)$ without AVC.

In this step, the cavity voltage is the superposition of the driving $\mathrm{rf}$ component $\left[V_{\mathrm{cav}, \mathrm{dr}}(h, t)\right]$ and the wake voltage $\left[V_{\text {cav, wake }}(h, t)\right]$. For the complex components for the harmonics $(h=2,4)$, the superposition is similar to (12) as

$$
V_{\text {cav }}(h, t)=H_{\mathrm{dr}}^{\text {cav }}(h, t) \cdot V_{\mathrm{dr}}(h, t)+Z_{\text {cav }}^{\prime}(h, t) \cdot I_{\text {beam }}(h, t),
$$

where $Z_{\text {cav }}^{\prime}(h, t)$ is the cavity gap impedance under the condition of the tube currents with the driving rf voltage and $I_{\text {beam }}(h, t)$ is the complex component of the beam current. By using the harmonic components $\left[V_{\text {cav }}(h, t)\right.$, $V_{\mathrm{dr}}(h, t)$, and $\left.I_{\text {beam }}(h, t)\right]$ and the transfer function obtained
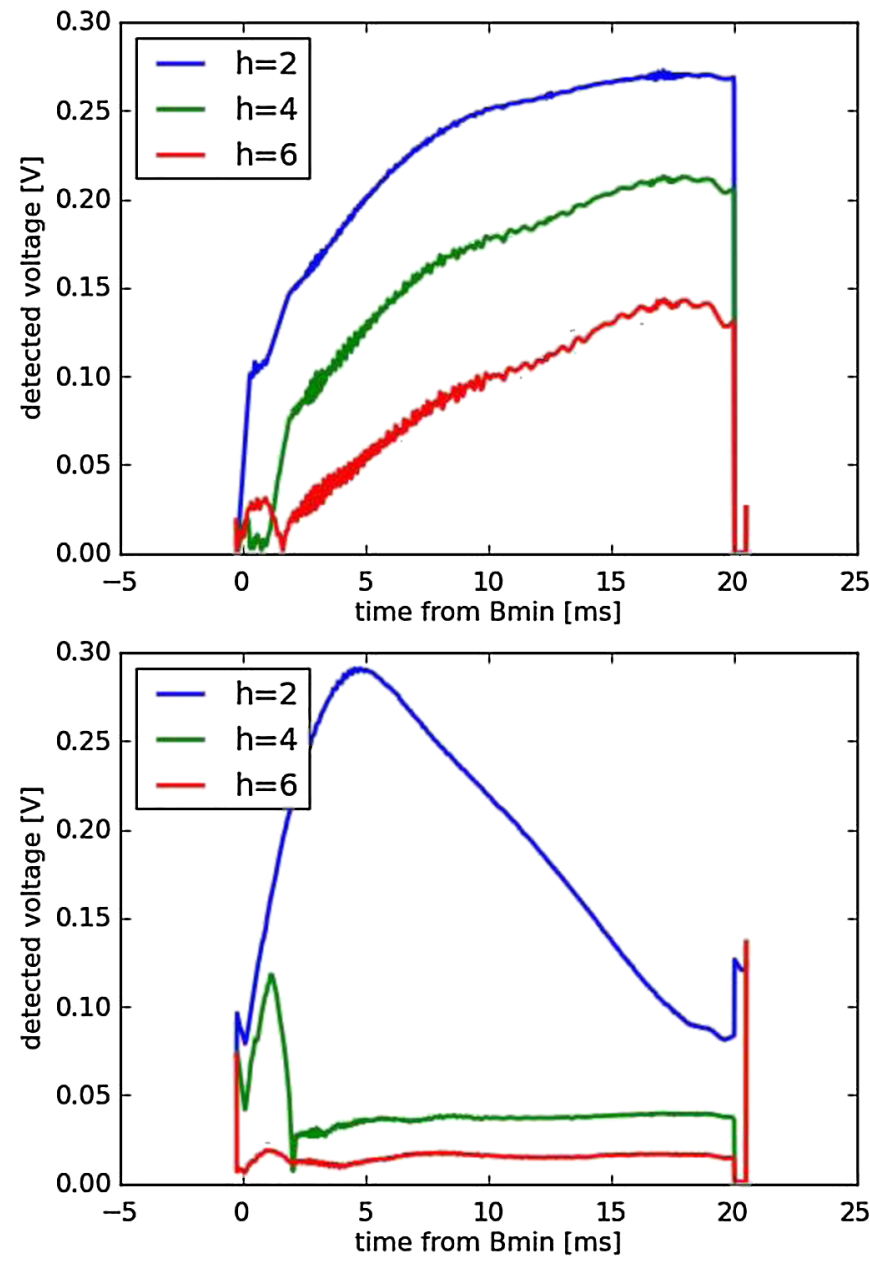

FIG. 13. Step 2 example (cavity \#1). The harmonic components of (top) the WCM and (bottom) the cavity voltage.

in step $1\left[H_{\mathrm{dr}}^{\mathrm{cav}}(h, t)\right]$, the cavity gap impedance $Z_{\mathrm{cav}}^{\prime}(h, t)$ is obtained.

\section{Step 3}

In this step, the feedforward compensation signal is applied to the target cavity.

The cavity voltage is the superposition of the driving $\mathrm{rf}$ component, the wake voltage, and the feedforward component $\left[V_{\text {cav, FF }}(h, t)\right]$, as follows:

$$
\begin{aligned}
V_{\mathrm{cav}}(h, t)= & H_{\mathrm{dr}}^{\mathrm{cav}}(h, t) \cdot V_{\mathrm{dr}}(h, t)+Z_{\mathrm{cav}}^{\prime}(h, t) \cdot I_{\text {beam }}(h, t) \\
& +Z_{\mathrm{FF}}(h, t) \cdot I_{\text {beam }}(h, t),
\end{aligned}
$$

where $Z_{\mathrm{FF}}(h, t)$ is the transfer function from the beam current to the feedforward voltage component.

By using the measured values $\left[V_{\text {cav }}(h, t), V_{\mathrm{dr}}(h, t)\right.$, and $\left.I_{\text {beam }}(h, t)\right]$ and the impedance and transfer functions $\left[H_{\mathrm{dr}}^{\mathrm{cav}}(h, t)\right.$ and $\left.Z_{\mathrm{cav}}^{\prime}(h, t)\right]$, the transfer function $Z_{\mathrm{FF}}(h, t)$ is obtained.

Similar to the case without driving rf voltage, the amplitude and phase patterns are modified so that the 
impedance seen by the beam, $Z_{\mathrm{cav}}^{\prime}(h, t)+Z_{\mathrm{FF}}(h, t)$, is minimized. For a given beam condition, only a few iterations of step 3 result in an impedance reduction in the order of $1 / 10$. For fine-tuning aiming to keep the impedance seen by the beam less than $25 \Omega$ during the full accelerating period, one or two additional iterations are necessary.

\section{Commissioning results}

The harmonic components of cavity \#1 gap monitor, without the feedforward and with the feedforward after the iterations of step 3, are shown in Fig. 14. The fundamental $(h=2)$ components are similar in both cases because the amplitude of the fundamental $\mathrm{rf}$ is strongly controlled by the AVC. The reduction of the second harmonic $(h=4)$ components is very clear after the program voltage becomes zero $(2 \mathrm{~ms})$. Since the pattern modification is not applied for the third harmonic $(h=6)$, the reduction of the $(h=6)$ wake voltage is limited.

The comparisons of the impedances and the feedforward transfer function of cavity $\# 1$ are shown in Fig. 15, both as function of time and in frequency domain.
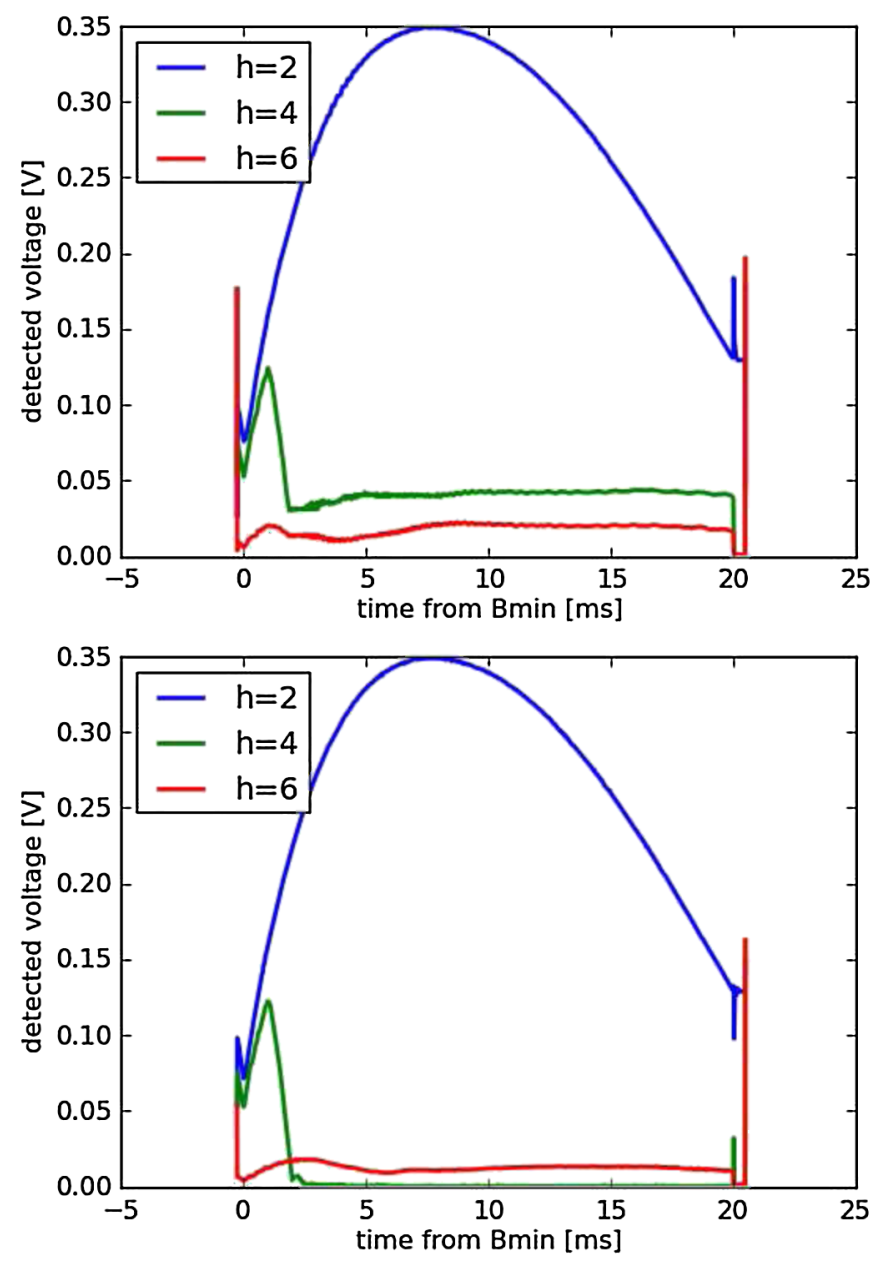

FIG. 14. The harmonic components of cavity \#1 gap monitor-(top) without the feedforward, (bottom) with the feedforward after the iterations of the step 3.
In the figure, "wake" indicates the impedance seen by the beam without feedforward, "FF component" indicates the transfer function from the beam current to the feedforward component of the cavity voltage, and "imp. seen by the beam" is the impedance seen by the beam with the feedforward signal after the iterations of step 3.

For the fundamental harmonic $(h=2)$ the amplitude of the feedforward component is very close to the impedance seen by the beam without feedforward from the beginning of the acceleration to the extraction. Thus, the impedance seen by the beam with feedforward is less than $25 \Omega$ while the shunt resistance of the accelerating gap is $800 \Omega$ during a full accelerating period.

In case of the second harmonic $(h=4)$, the impedance reduction is limited until $2.5 \mathrm{~ms}$ due to the error of the feedforward transfer function. However, after $2.5 \mathrm{~ms}$ the reduction of the impedance seen by the beam is fine, it is less than $25 \Omega$.
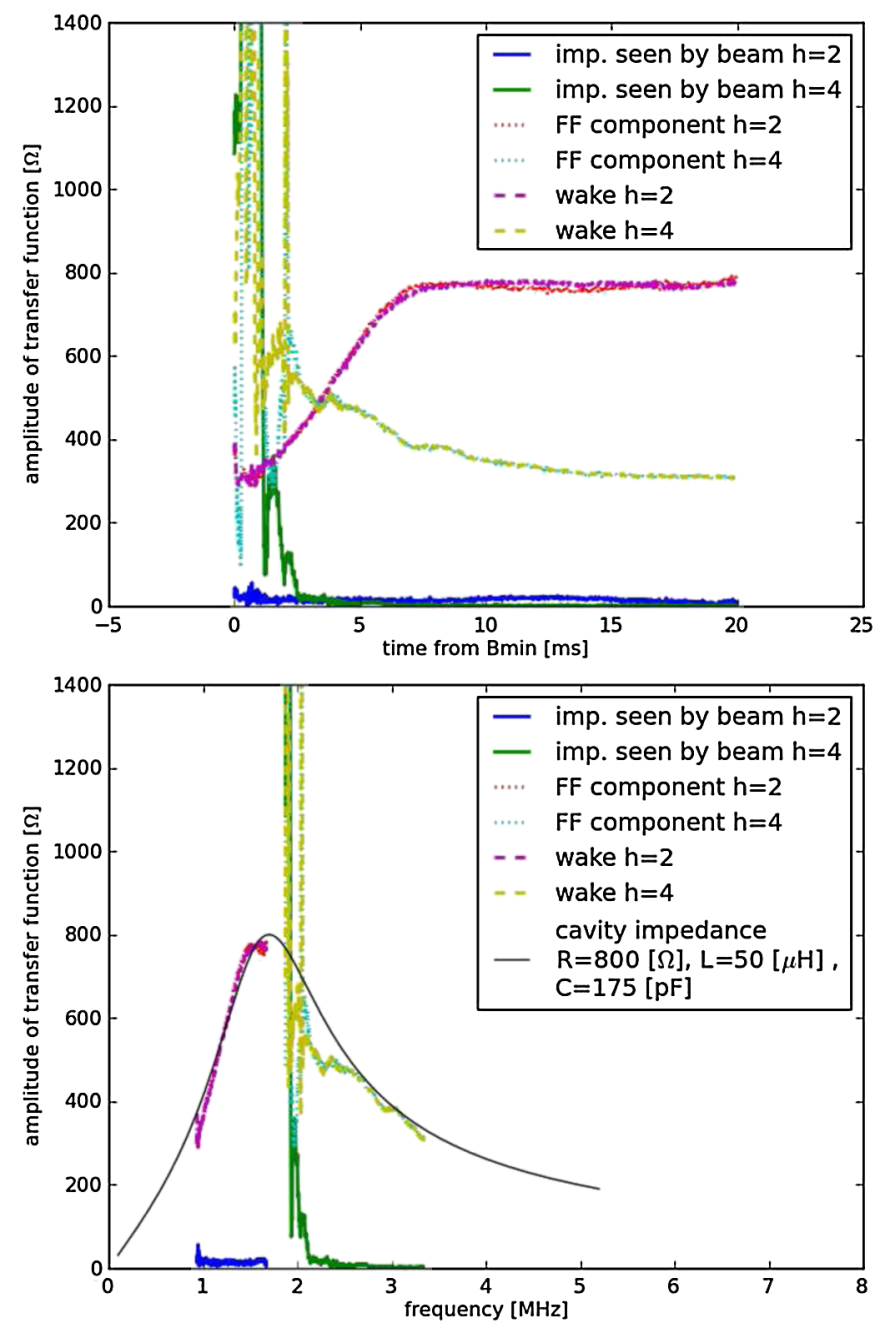

FIG. 15. The comparison of the impedances and the feedforward transfer function of cavity\#1-(top) as function of time, (bottom) translation to frequency domain. In the frequency domain, the impedance of a parallel LCR circuit is also plotted. 
A comparison of the cavity voltage waveform with no beam, with the $300 \mathrm{~kW}$ equivalent beam without feedforward, and with feedforward, is shown in Fig. 16. In the figure the waveforms at the middle of the acceleration $(10 \mathrm{~ms})$ and just before extraction $(20 \mathrm{~ms})$ are plotted. The waveforms are sinusoidal without the beam because the second harmonic program voltage is zero at the middle and end of the acceleration. Without feedforward, the voltage distortion is observed due to the higher harmonic wake voltages. Also, one can notice the delay of the waveform compared to the case of no beam, which is corresponding to the loading angle. With feedforward, the distortion is fairly reduced and the phase shift of the waveform is much smaller.

A phasor diagram of the voltage and currents with the feedforward compensation is shown in Fig. 17. In the figure, $I_{b}$ is the beam current, $I_{g}$ (total) is the total generator current that consists of the driving rf component $I_{g}$ (drive)
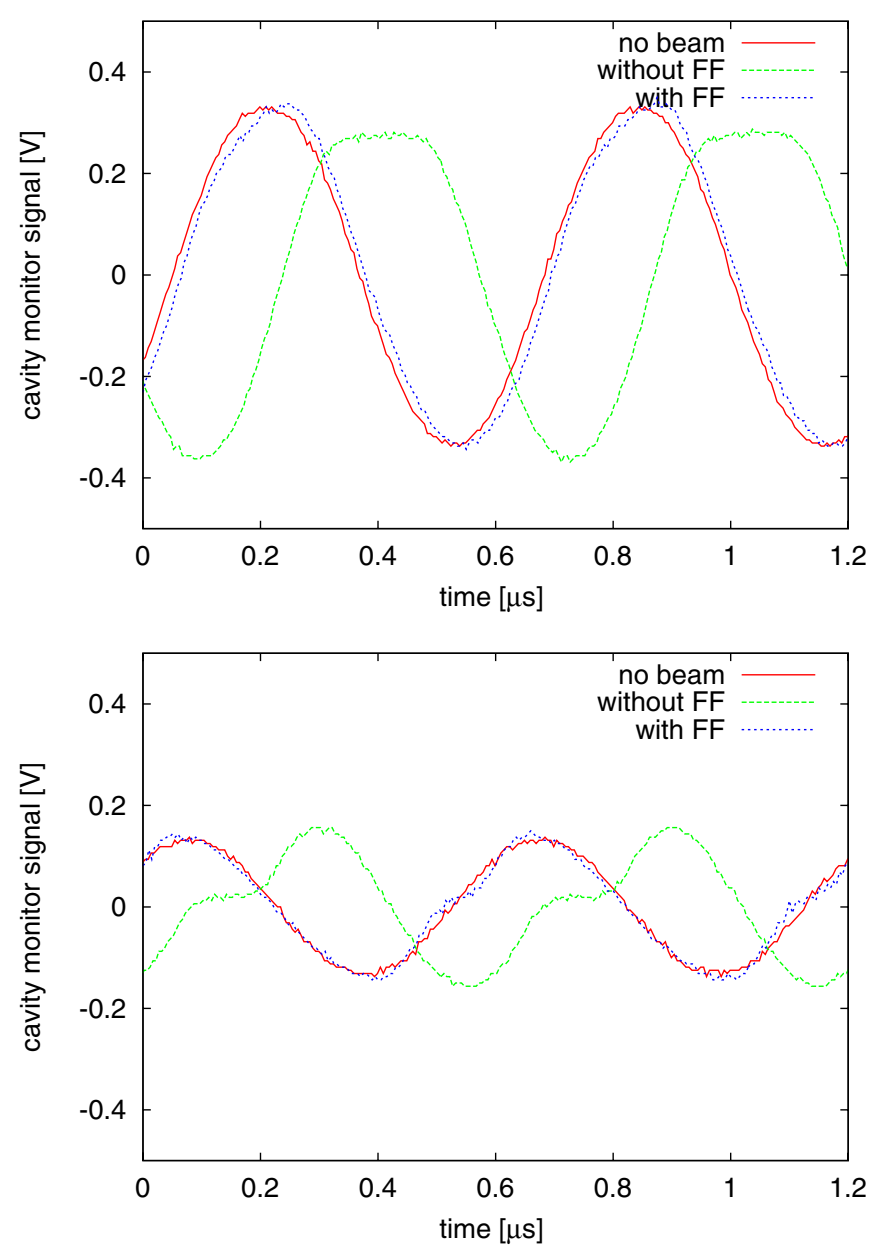

FIG. 16. Comparisons of cavity \#1 voltage monitor waveforms in the cases of no beam, without feedforward, with feedforward. The beam intensity is $300 \mathrm{~kW}$ equivalent-(top) middle of acceleration period and (bottom) just before extraction. The distortion of the voltage waveform is reduced with feedforward.

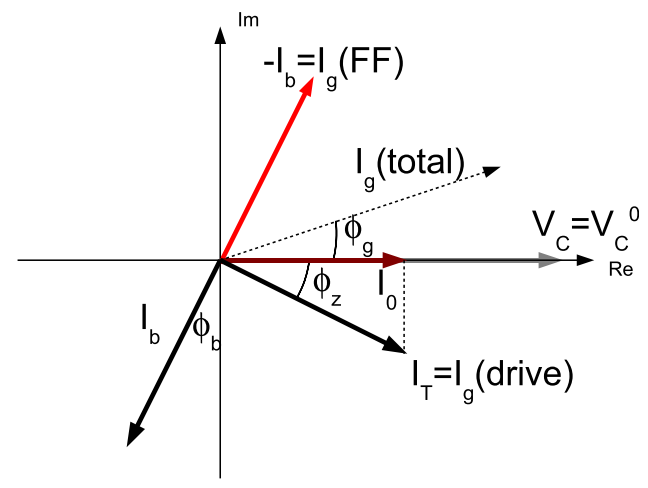

FIG. 17. Phasor diagram with the feedforward compensation.

and the feedforward component $I_{g}(\mathrm{FF})$, and $I_{T}$ is the total current fed into the cavity. The phases $\phi_{b}, \phi_{z}$, and $\phi_{g}$ are the beam phase, the detuning angle of the cavity, and the loading angle, respectively. If the feedforward compensates the beam loading perfectly, $I_{g}$ (drive) is same as the generator current without the beam, which generates the voltage $V_{c}^{0}$.

The amplitudes of the LLRF driving $\mathrm{rf}$ in the cases of no beam, with beam and no feedforward, and with beam and feedforward, are plotted in Fig. 18. Without the feedforward, the voltage control loop increases the driving if amplitude to keep the voltage to against the beam loading. On the other hand, the driving rf amplitude with the feedforward is very close to the case of no beam. This indicates that the beam loading compensation is working properly and the condition $I_{T}=I_{g}$ (drive) is satisfied.

All measurements above confirm that the beam loading compensation by the rf feedforward system works satisfactorily. The commissioning of the feedforward for 11

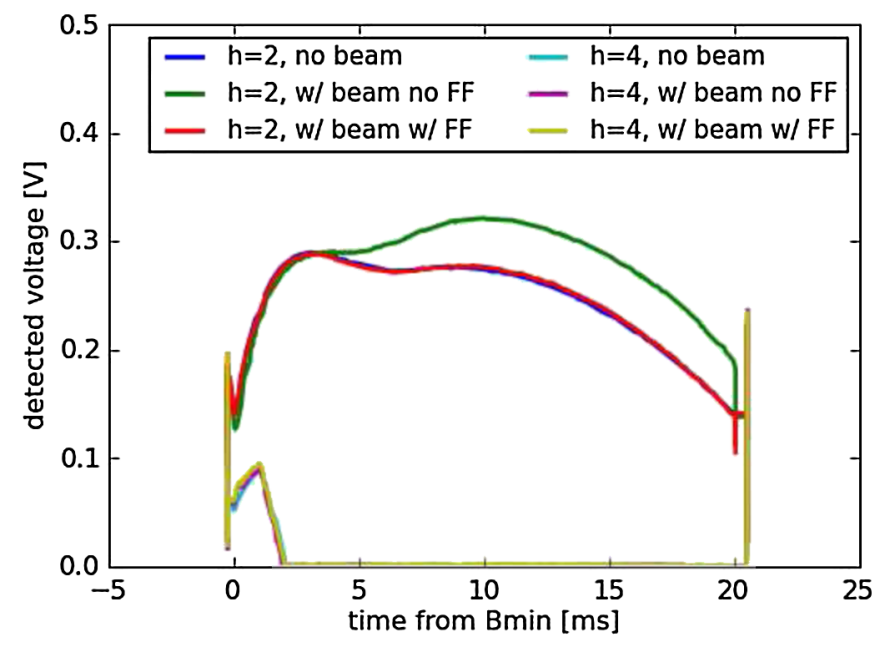

FIG. 18. A comparison of the driving rf voltage of no beam, without and with the feedforward. The cases of no beam and with feedforward are very close. 
cavities has beam performed and the feedforward is used in routine operations of the RCS.

\section{IMPROVEMENTS OF THE BEAM QUALITY WITH THE FEEDFORWARD}

The distortion of the cavity voltage waveform by the higher harmonic components of the wake voltage results in a synchronous phase $\left(\phi_{s}\right)$ deviation, because the energy gain per turn at the synchronous phase must be the same in both cases of undistorted and distorted waveforms. In Fig. 19 the comparison of $\phi_{s}$ in an accelerating period of the $300 \mathrm{~kW}$ equivalent beams without and with the feedforward is shown. In case of the feedforward, the synchronous phases with various beam power are also plotted. One can see that the synchronous phase is smaller with the feedforward because the voltage distortion is fairly reduced, as shown in Fig. 16. The maximum $\phi_{s}$ is reduced from 55 degrees to 49 degrees with the feedforward. The dependency of the synchronous phase on the beam intensity is quite small with the feedforward.

The rf buckets shrink with higher $\phi_{s}$, which may cause beam losses at the high dispersion points in the arc section. A comparison of beam loss monitor signals at the dispersion peak in the arc is shown in Fig. 20. Although the intensity loss is not observed by the DC current transformer, there is a small amount of losses at the dispersion peak in the middle of the acceleration and the losses are possibly a cause of the radioactivation in the arc sections. On the other hand, the losses disappear with the feedforward, which reduces voltage distortion and keeps the margin of the rf bucket.

Beams with larger longitudinal emittance can be accelerated by the wider rf bucket with the feedforward, and we have more choices of the longitudinal painting parameters, for example, the momentum offset and the duration of the

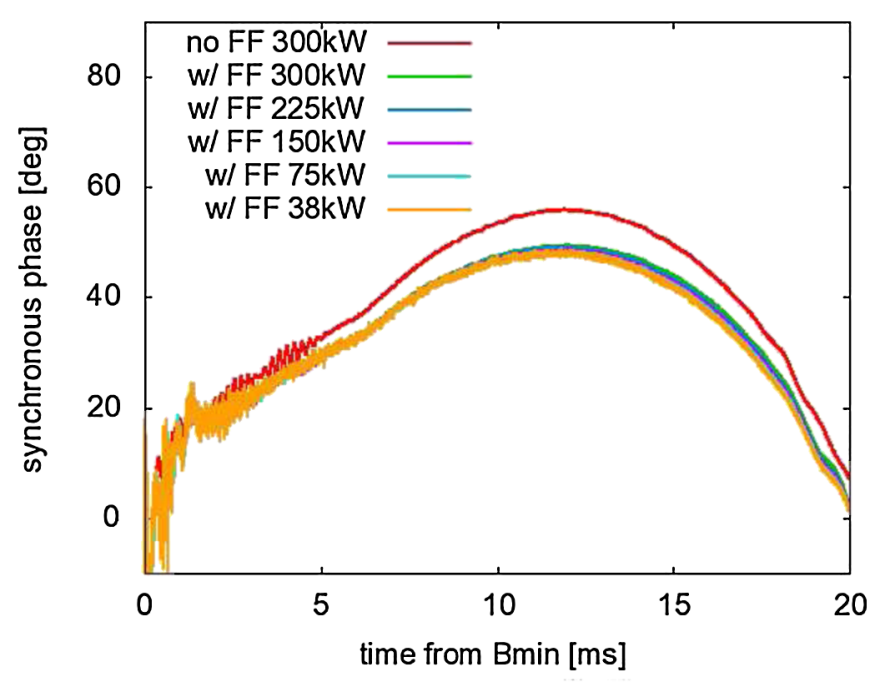

FIG. 19. $\phi_{s}$ comparison with and without the feedforward.

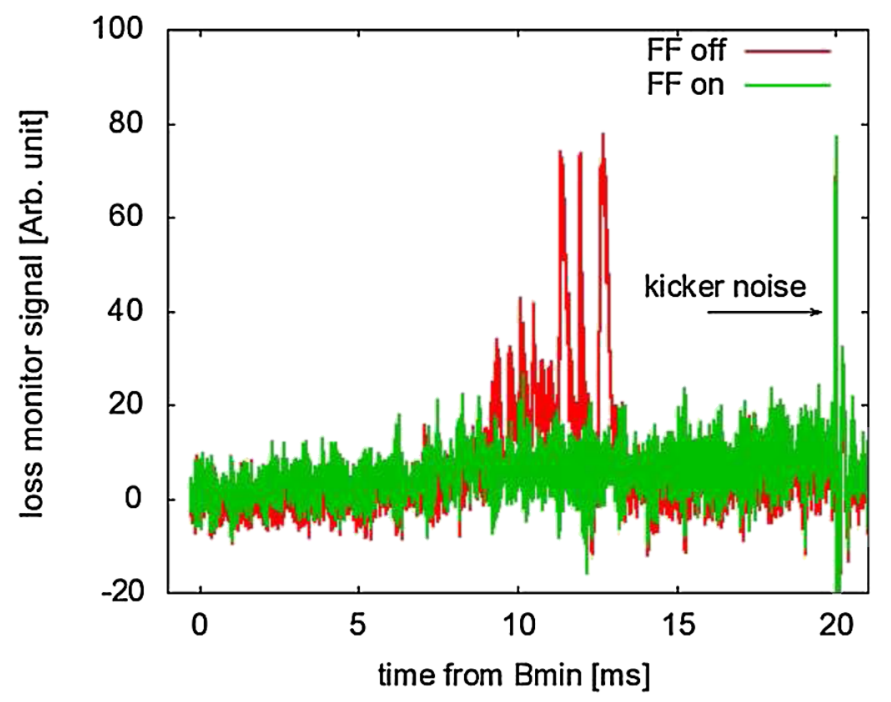

FIG. 20. Beam loss monitor signals at the arc section with the accelerating $300 \mathrm{~kW}$ equivalent beams. The beam loss is reduced by the feedforward.
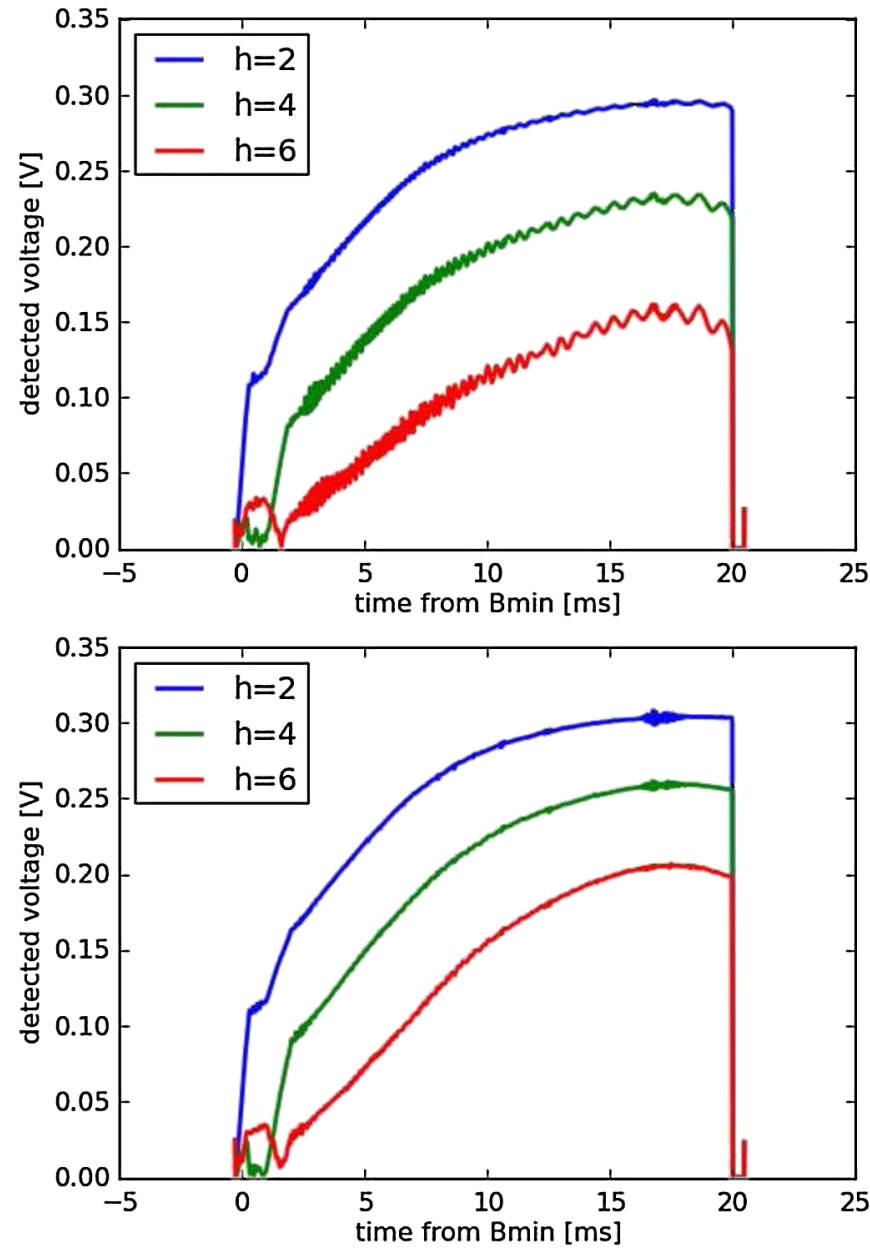

FIG. 21. Harmonic components of the WCM beam signals (top) without and (bottom) with the feedforward. The oscillation of harmonic components is much reduced. 
second harmonic voltages, which may result in larger longitudinal emittance.

The rf voltage pattern of the RCS is programmed so that the momentum filling factor is kept until the middle of the acceleration to avoid unwanted emittance blowups. However, the rf buckets are not as programmed if the voltage waveforms are distorted. In Fig. 21 the harmonic components $(h=2,4,6)$ of the WCM beam signal of the $300 \mathrm{~kW}$ equivalent beam with and without feedforward are plotted. Without the feedforward, the harmonic components start oscillations from $3 \mathrm{~ms}$, which is corresponding to the quadrupole oscillation of the beam due to the mismatch by the voltage waveform distortions. With the feedforward, the oscillations of the harmonics are very small. This means that the feedforward compensation greatly reduces the voltage waveform distortion and the beam feels the accelerating voltage as programmed.

The bunch oscillation causes the unwanted longitudinal emittance blowup. In Fig. 22, the bunch shapes just before the extraction without and with the feedforward are shown. Without the feedforward compensation, the bunch is wider
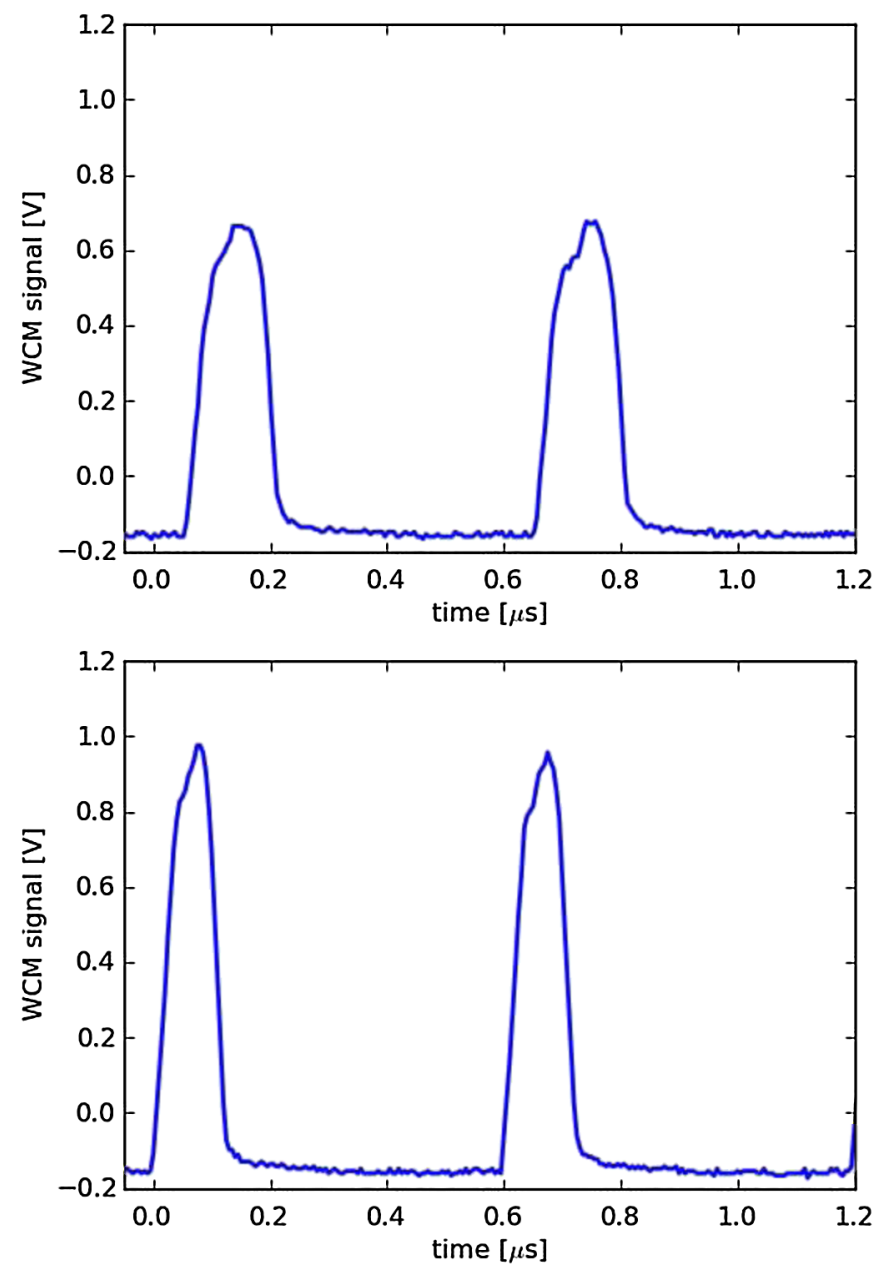

FIG. 22. Bunch shapes of just before extraction (top) without and (bottom) with the feedforward. because of the unwanted emittance blowup. With the feedforward compensation, the longitudinal beam emittance is as programmed and the bunch is narrower. Therefore, the control of the extracted bunch shape is possible with the feedforward compensation.

\section{SCALABILITY AND STABILITY OF THE FEEDFORWARD}

Because of its open-loop setup, the performance of the feedforward may degrade with drifts or changes of any elements of the rf system. If all parts of the rf system were linear, the performance of the feedforward would be independent of the beam intensity. However, the tube current, on which the tube gain depends, is a function of the accelerating beam current. Therefore, we checked the dependency of the feedforward performance on the beam intensity.
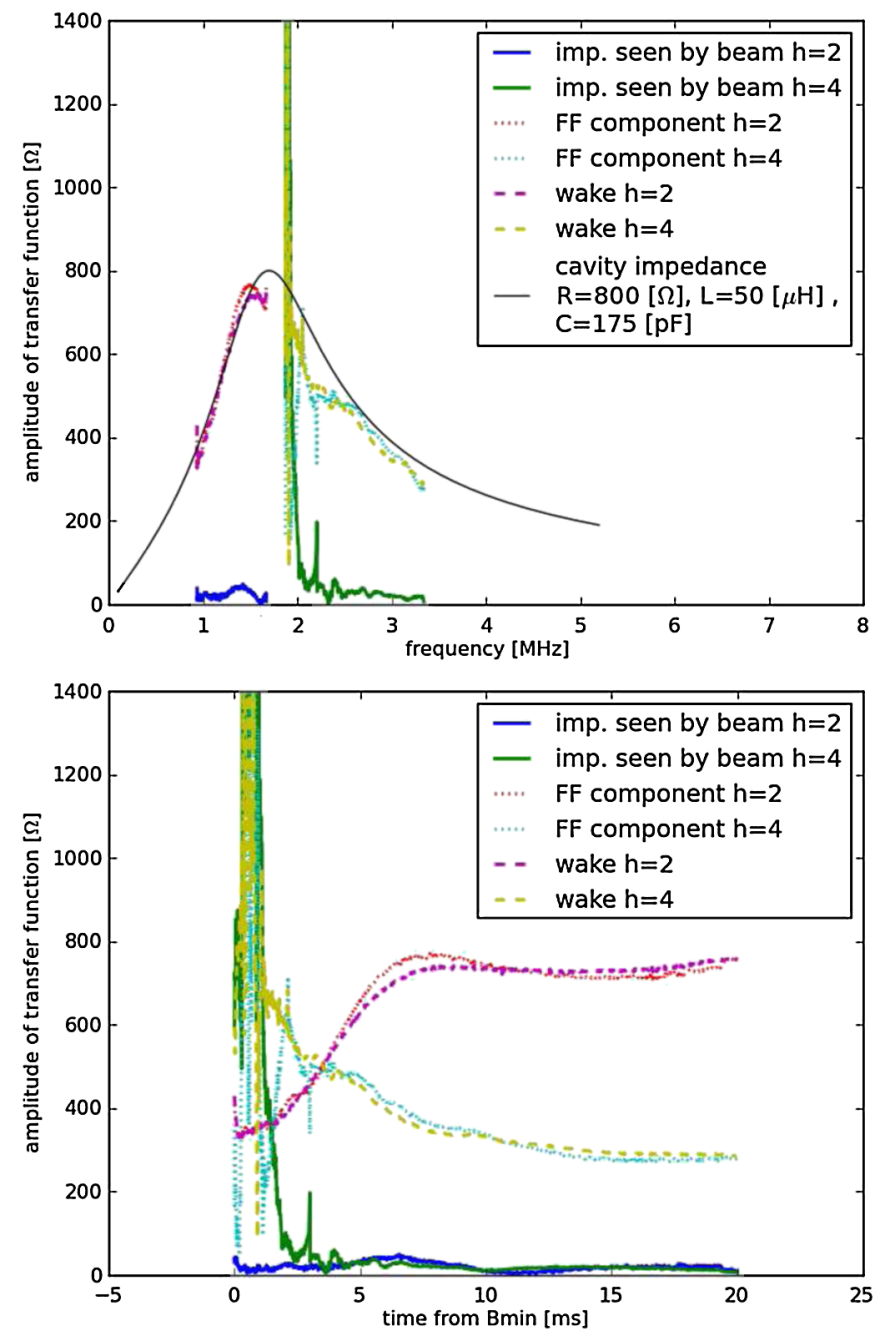

FIG. 23. Cavity \#11 impedance seen by the $400 \mathrm{~kW}$ equivalent beam with the feedforward parameters optimized by the $300 \mathrm{~kW}$ equivalent beam-(top) frequency domain and (bottom) as a function of time. 
We used cavity \#11 in the test. The feedforward patterns of the cavity are optimized by using the $300 \mathrm{~kW}$ equivalent beam $\left(2.5 \times 10^{13} \mathrm{ppp}\right)$. We accelerated the test beams at the intensity of $400 \mathrm{~kW}$ equivalent $\left(3.3 \times 10^{13} \mathrm{ppp}\right)$ and measured the impedance seen by the beam by using steps 1-3 described in the previous section.

The measurement results are plotted in Fig. 23. Clearly we notice that there are some errors of the feedforward amplitude and the reduction of the impedance seen by the beam is not perfect as in the $300 \mathrm{~kW}$ case. However, the error is small and the reduction is better than $1 / 14$, the worst point is around $7 \mathrm{~ms}$, where the impedance is reduced from 700 to $50 \Omega$. Therefore, it is confirmed that the feedforward patterns optimized by the $300 \mathrm{~kW}$ equivalent beam can be used for higher beam intensities, at least $30 \%$ more intensity, $400 \mathrm{~kW}$. The performance degradation by the intensity change seems small.
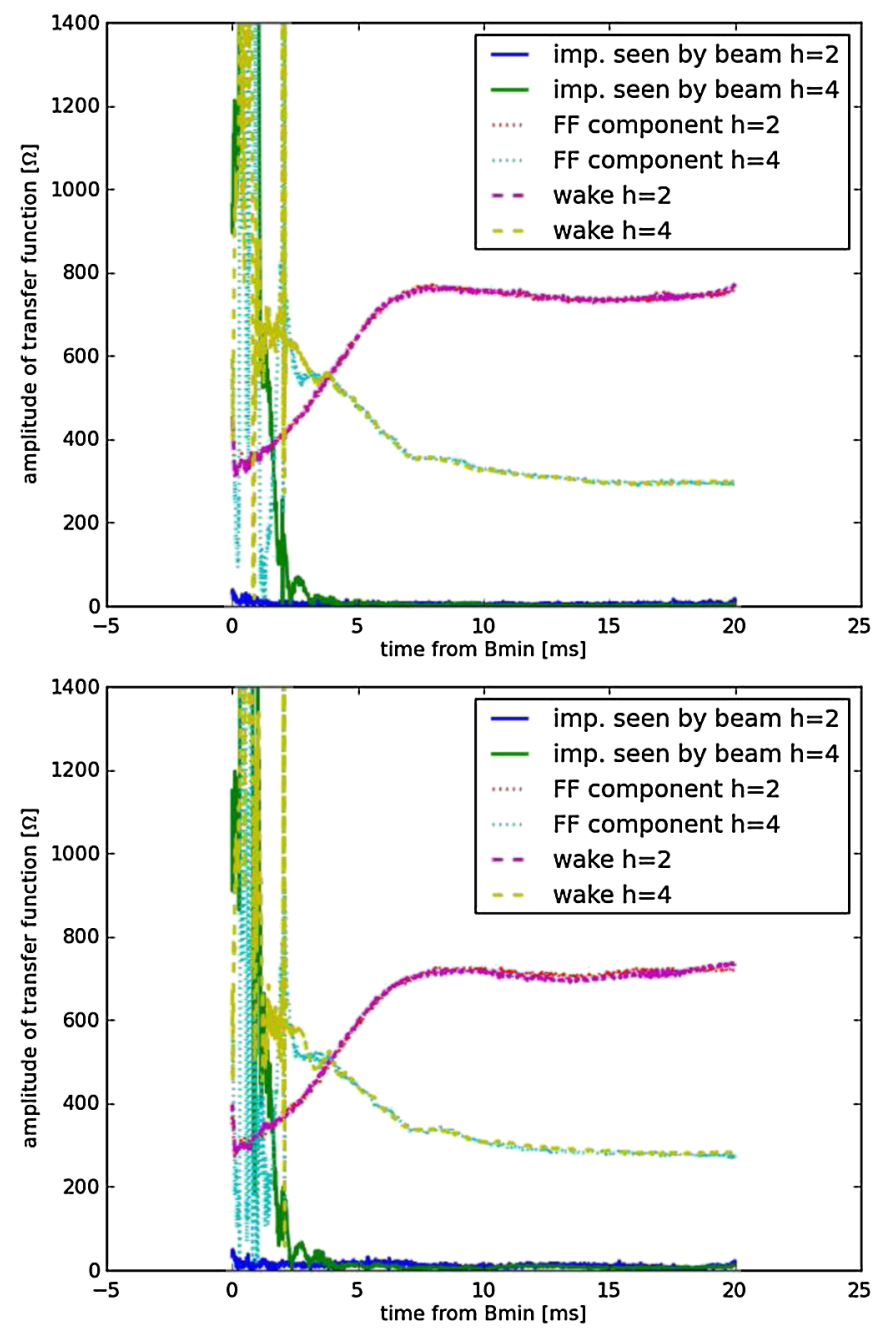

FIG. 24. Cavity \#2 impedance seen by the beam as function of time-(top) just after the feedforward patterns are optimized in June 2010 and (bottom) as of March 2011. The impedance seen by the beam is still low. It was not necessary to optimize the patterns again.
We also performed the pattern optimization of the \#11 system by steps $1-3$ with the $400 \mathrm{~kW}$ equivalent beam. The impedance seen by the beam was successfully reduced. Hence, we confirmed that the commissioning methodology of the feedforward works with the higher beam power.

The feedforward patterns of all 11 cavities were commissioned in June 2010, and it was not necessary to optimize again until the present time of writing this article, March 2011, except for the cases where the cavity configuration changed (the \#1, \#4, and \#5 systems). A comparison of impedance seen by the beam of cavity \#2 between just after optimization in June 2010 and as of March 2010, is shown in Fig. 24. One can see that a good impedance reduction is still achieved in the case of March 2011. This is not only because of the stability of the full-digital feedforward system, but also because the high power rf system including the MA cavities is stable.

\section{SUMMARY AND DISCUSSIONS}

We summarize the article as follows.

1. For the high intensity operation of the J-PARC RCS, a multiharmonic beam loading compensation, which cancels the wake voltages of not only the fundamental accelerating rf but also the higher harmonics in the wide-band MA cavities, is necessary.

2. The rf feedforward method is employed for the beam loading compensation in the RCS.

3. A multiharmonic feedforward compensation system is developed as a part of the full-digital LLRF control system. The feedforward system can cancel the most important three harmonics $(h=2,4,6)$. The system works as a bandpass filter with the passbands of the harmonics ( $h=2,4,6)$, whose passband follows the accelerating frequency sweep. The gain and phase patterns of the feedforward are programmed to cancel the wake voltages.

4. The commissioning methodologies of the feedforward, in both cases without and with the driving rf voltages, are established. The commissioning has been performed with the $300 \mathrm{~kW}$ equivalent beams and the commissioning of the feedforward systems for all 11 cavities of the RCS was successfully done.

5. The beam loss in the high dispersion area is reduced by the feedforward. Also, the unwanted longitudinal emittance blowup is reduced. The beam sees the accelerating voltage as programmed.

6. The scalability and the stability of the beam loading compensation by the feedforward has been confirmed.

In the commissioning of the feedforward, we assume the reproducibility of the transfer functions and cavity impedances. The reproducibility is assured by the following items: The MA cavity without the tuning bias loop can be a passive load. In the case of the ferrite cavities with the tuning bias loop, the cavity impedance varies as the tuning loop control makes errors. The timing system of the J-PARC is not synchronized to the AC-line frequency. 
The J-PARC accelerators work by the master clock and the master trigger, which is based on the high-quality synthesizer. Since the accelerator cycle is fixed independently of the AC-line frequency drifts and the DDS generates a very precise frequency, the rf frequency pattern of the RCS can be fixed without a radial loop. We emphasize that the reproducibility of the J-PARC RCS finally resulted in a good reduction of the impedance seen by the beam with the multiharmonic feedforward compensation system.

We are considering to apply the feedforward compensation of the odd harmonics $(h=1,3,5)$ to accelerate a single bunch in the RCS. The periodic transient beam loading by the single bunch acceleration is limiting the bunch intensity. The maximum particles per bunch is less than that of the two bunch operation. By compensating the odd harmonics we should be able to accelerate a bunch with protons, which is comparable to the two bunch operation. The high intensity single bunch acceleration will be beneficial for some of the neutron and muon experiments. A preliminary beam test for the compensation of the odd harmonics has been started.

To conclude the article, we emphasize that the performance of the multiharmonic feedforward is encouraging the high power operation at the design intensity.

\section{ACKNOWLEDGMENTS}

We would like to thank Professor E. Ezura, Professor K. Takata, Professor E. Takasaki, and Professor A. Ando for fruitful discussions and comments. We are grateful to the J-PARC RCS commissioning group headed by H. Hotchi for giving us the beam time for the commissioning of the rf feedforward. Also, we would like to thank all the J-PARC RCS members. The facilities of J-PARC were severely damaged by the Tohoku earthquake on March 11, 2011. We appreciate the accelerator communities in the world for their concerns and support. We will do our best for the recovery of the machine, and we will make our best effort to achieve the design intensity after the recovery.

[1] JHF Project Office, KEK Report No. 97-16, 1997.

[2] High-Intensity Proton Accelerator Project Team, JAERI-TECH Report No. 2003-044, 2003.
[3] H. Hotchi et al., Phys. Rev. ST Accel. Beams 12, 040402 (2009).

[4] F. Tamura et al., Phys. Rev. ST Accel. Beams 12, 041001 (2009).

[5] H. Hotchi et al., in Proceedings of the First International Particle Accelerator Conference (IPAC 10) (2010), pp. 624-626.

[6] F. Pedersen, IEEE Trans. Nucl. Sci. 22, 1906 (1975).

[7] M. Yamamoto et al., in Proceedings of the First International Particle Accelerator Conference (IPAC 10) (2010), pp. 1940-1942.

[8] E. Ezura et al., IEEE Trans. Nucl. Sci. 26, 3538 (1979).

[9] S. Ninomiya et al., Part. Accel. 32, 69 (1990) [http:// cdsweb.cern.ch/record/1108194].

[10] F. Pedersen, IEEE Trans. Nucl. Sci. 32, 2138 (1985).

[11] R. Garoby et al., in Proceedings of the 1989 Particle Accelerator Conference, Chicago, IL (IEEE, New York, 1989), pp. 135-137.

[12] P. Barratt et al., in Proceedings of the 2nd European Particle Accelerator Conference (EPS and ECFA, Nice, 1990), pp. 949-951.

[13] M. Yamamoto et al., in Proceedings of the 18th Particle Accelerator Conference, New York, 1999 (IEEE, New York, 1999), pp. 860-862.

[14] M. Fujieda et al., in Proceedings of the 18th Particle Accelerator Conference, New York, 1999 (Ref. [13]), pp. 857-859.

[15] R. Garoby, in Proceedings of the U.S.-CERN-Japan International School (World Scientific, Hayama and Tsukuba, 1996) pp. 455-489.

[16] F. Tamura et al., in Proceedings of the 2005 Particle Accelerator Conference (IEEE, Knoxville, 2005), pp. 3624-3626.

[17] F. Tamura et al., in Proceedings of the 2005 Particle Accelerator Conference (Ref. [16]), pp. 3853-3855.

[18] F. Tamura et al., Phys. Rev. ST Accel. Beams 11, 072001 (2008).

[19] F. Tamura et al., in Proceedings of 11th European Particle Accelerator Conference (EPAC 2008) (EPS-AG, Genoa, Italy, 2008), pp. 364-366.

[20] F. Tamura et al., in Proceedings of the first International Particle Accelerator Conference (IPAC 10) (2010), pp. 1143-1145.

[21] F. Tamura et al., in Proceedings of the 10th European Particle Accelerator Conference, Edinburgh, Scotland, 2006 (EPS-AG, Edinburgh, Scotland, 2006), pp. 1319-1321. 